\title{
Enantioselective Synthesis of Cyclic Enol Ethers and Formation of All-Carbon Quaternary Stereogenic Centers through Catalytic Asymmetric Ring-Closing Metathesis (ARCM)
}

\author{
Ai-Lan Lee, ${ }^{\ddagger}$ Steven J. Malcolmson, ${ }^{\ddagger}$ Alessandra Puglisi, ${ }^{,}$Richard R. Schrock,,${ }^{\dagger}$ \\ and Amir H. Hoveyda ${ }^{\ddagger, *}$ \\ Department of Chemistry, Merkert Chemistry Center, Boston College \\ Chestnut Hill, Massachusetts 02467 \\ Department of Chemistry, Massachusetts Institute of Technology \\ Cambridge, Massachusetts 02139
}

\section{SUPPORTING INFORMATION}

General. All reactions were carried out in oven- $\left(135^{\circ} \mathrm{C}\right)$ or flame-dried glassware under an inert atmosphere of dry $\mathrm{N}_{2}$ unless otherwise stated. Infrared (IR) spectra were recorded on a Nicolet 210 spectrophometer, $v_{\max }$ in $\mathrm{cm}^{-1}$. Bands are characterized as broad (br), strong (s), medium (m) or weak (w). ${ }^{1} \mathrm{H}$ NMR spectra were recorded on a Varian Gemini 2000 (400 $\mathrm{MHz}$ ) spectrometer. Chemical shifts are reported in ppm from tetramethylsilane with the solvent resonance resulting from incomplete deuteration as the internal reference $\left(\mathrm{CDCl}_{3}: \delta\right.$ 7.26, $\left.\mathrm{C}_{6} \mathrm{D}_{6}: \delta 7.16\right)$. Data are reported as follows: chemical shift, multiplicity ( $\mathrm{s}=$ singlet, $\mathrm{d}=$ doublet, $\mathrm{t}=$ triplet, $\mathrm{q}=$ quartet, $\mathrm{br}=$ broad, $\mathrm{m}=$ multiplet $)$, coupling constants $(\mathrm{Hz})$, integration and assignment. ${ }^{13} \mathrm{C}$ NMR spectra were recorded on a Varian Gemini $2000(100 \mathrm{MHz})$ spectrometer with complete proton decoupling. Chemical shifts are reported in ppm from tetramethylsilane with the solvent as the internal reference $\left(\mathrm{CDCl}_{3}: \delta 77.16, \mathrm{C}_{6} \mathrm{D}_{6}: \delta 128.06\right)$. Enantiomer ratios were determined by chiral HPLC (Chiral Technologies Chiralpak OD column (4.6 mm x $250 \mathrm{~mm}$ )) or by chiral GLC analysis (Alltech Associates Chiraldex GTA or $\mathrm{BPH}$, Supelco Betadex or Alphadex 120 column $(30 \mathrm{~m} \times 0.25 \mathrm{~mm})$ ) in comparison with authentic racemic materials. High-resolution mass spectrometry was performed at the University of Illinois Mass Spectrometry Laboratory. Optical rotation values were recorded on a Rudolph Research Analytical Autopol IV polarimeter. Melting points were measured on a Thomas Hoover capillary melting point apparatus and are uncorrected. X-ray crystal structure was determined at the Harvard University X-Ray Crystallographic Laboratory. 
Materials. Solvents were purged with argon and purified under a positive pressure of dry argon by a modified Innovative Technologies purification system: $\mathrm{Et}_{2} \mathrm{O}$ and $\mathrm{CH}_{2} \mathrm{Cl}_{2}$ were passed through activated alumina columns; benzene, toluene and pentane ${ }^{1}$ were passed successively through activated $\mathrm{Cu}$ and alumina columns. THF was distilled from sodium benzophenone ketyl. Unless otherwise stated, commercially available reagents were used as received. Mo-based complexes 1, 3a-c, 4a-c, 5, and $\mathbf{6}$ were prepared according to published procedures. $^{2}$ Mo complexes were handled under an inert atmosphere in a dry box. Ru-based complex 2 was purchased from Materia and purified by silica gel chromatography and recrystallization prior to use. ${ }^{3}$ Grubbs's second-generation catalyst $\mathbf{1 1}$ was purchased from Materia and purified by silica gel chromatography prior to use. Ru-based complexes $\mathbf{7} \mathbf{a}-\mathbf{b}^{4}$ and $\mathbf{8 a}-\mathbf{b}^{5}$ were prepared according to published procedures. Ru-based complexes were handled under an inert atmosphere in a dry box for comparison purposes.

The vinyl ether substrates were prepared from the corresponding alcohols using the Pdvinyl transfer method developed by Bosch and Schlaf. 6 The resulting enol ethers were purified by silica gel chromatography (eluted with $1 \mathrm{~mol}_{\%} \mathrm{Et}_{3} \mathrm{~N}$ ).

2,8-Dimethyl-5-(vinyloxy)nona-1,8-diene (10). IR (neat): 2936 (s), 1652 (s), 1627 (s), 1608 (m), 1375 (m), 1325 (w), 1193 (s), 1162 (s), 1073 (w), 885 (s), 809 (m) cm ${ }^{-1}$. ${ }^{1} \mathrm{H}$ NMR (400 $\left.\mathrm{MHz}, \mathrm{CDCl}_{3}\right): \delta 6.28\left(\mathrm{dd}, J=14.0,6.4 \mathrm{~Hz}, 1 \mathrm{H}, \mathrm{OC} H=\mathrm{CH}_{2}\right), 4.71\left(\mathrm{~s}, 2 \mathrm{H}, \mathrm{CH}_{3} \mathrm{C}=\mathrm{CH} H^{\prime}\right), 4.67$ (s, 2H, $\left.\mathrm{CH}_{3} \mathrm{C}=\mathrm{CHH}^{\prime}\right), 4.26\left(\mathrm{~d}, J=14.0 \mathrm{~Hz}, 1 \mathrm{H}, \mathrm{OCH}=\mathrm{CH} H^{\prime}\right), 3.94(\mathrm{~d}, J=6.4 \mathrm{~Hz}, 1 \mathrm{H}$, $\left.\mathrm{OCH}=\mathrm{CH} \mathrm{H}^{\prime}\right), 3.71(\mathrm{tt}, J=6.4,6.4 \mathrm{~Hz}, 1 \mathrm{H}, \mathrm{CHO}), 2.15-2.03\left(\mathrm{~m}, 4 \mathrm{H}, \mathrm{CH}_{2}\right), 1.80-1.60(\mathrm{~m}, 4 \mathrm{H}$, $\left.\mathrm{CH}_{2}\right), 1.71\left(\mathrm{~s}, 6 \mathrm{H}, \mathrm{CH}_{3}\right) .{ }^{13} \mathrm{C} \mathrm{NMR}\left(100 \mathrm{MHz}, \mathrm{CDCl}_{3}\right): \delta 151.9,145.5,110.3,88.0,79.5,33.5$, 32.4, 22.7. HRMS calcd for $\mathrm{C}_{13} \mathrm{H}_{22} \mathrm{O}: 194.1671$. Found: 194.1665.

2,6-Dimethyl-4-(vinyloxy)hepta-1,6-diene (12). IR (neat): 3071 (w), 2971 (m), 2940 (m), 1649 (m), 1633 (s), 1609 (m), 1448 (w), 1379 (w), 1354 (w), 1324 (w), 1120 (s), 1175 (s), $1070(\mathrm{~m}), 890$ (s), $816(\mathrm{w}), 741(\mathrm{w}) \mathrm{cm}^{-1} .{ }^{1} \mathrm{H} \mathrm{NMR}\left(400 \mathrm{MHz}, \mathrm{CDCl}_{3}\right): \delta 6.30$ (dd, $J=14.4$, $\left.6.8 \mathrm{~Hz}, 1 \mathrm{H}, \mathrm{OCH}=\mathrm{CH}_{2}\right), 4.81\left(\mathrm{~s}, 2 \mathrm{H}, \mathrm{CH}_{3} \mathrm{C}=\mathrm{CH} H^{\prime}\right), 4.73\left(\mathrm{~s}, 2 \mathrm{H}, \mathrm{CH}_{3} \mathrm{C}=\mathrm{CHH}^{\prime}\right), 4.27$ (dd, $J=$

1) $n$-Pentane was allowed to stir over concentrated $\mathrm{H}_{2} \mathrm{SO}_{4}$ for five days, washed with water, followed by a saturated aqueous solution of $\mathrm{NaHCO}_{3}$, dried over $\mathrm{MgSO}_{4}$, and filtered before use in the solvent purification system.

2) R. R. Schrock, A. H. Hoveyda, Angew. Chem. Int. Ed. 2003, 42, 4555-4708 and references cited therein.

3) S. B. Garber, J. S. Kingsbury, B. L. Gray, A. H. Hoveyda, J. Am. Chem. Soc. 2000, 122, 8168-8179.

4) J. J. Van Veldhuizen, D. G. Gillingham, S. B. Garber, O. Kataoka, A. H. Hoveyda, J. Am. Chem. Soc. 2003, $125,12502-12508$.

5) J. J. Van Veldhuizen, J. E. Campbell, R. E. Giudici, A. H. Hoveyda, J. Am. Chem. Soc. 2005, 127, 6877-6882.

6) M. Bosch, M. Schlaf, J. Org. Chem. 2003, 68, 5225-5227. 
14.4, 1.6 Hz, 1H, OCH=CHH'), $4.07(\mathrm{tt}, J=6.8,5.6 \mathrm{~Hz}, 1 \mathrm{H}, \mathrm{CHO}), 3.97(\mathrm{dd}, J=6.8,1.6 \mathrm{~Hz}$, $\left.1 \mathrm{H}, \mathrm{OCH}=\mathrm{CH} \mathrm{H}^{\prime}\right), 2.30\left(\mathrm{dd}, J=14.4,6.8 \mathrm{~Hz}, 2 \mathrm{H}, \mathrm{OCHCH} H^{\prime}\right), 2.21(\mathrm{dd}, J=14.4,5.6 \mathrm{~Hz}, 2 \mathrm{H}$, OCHCHH'), 1.75 (s, 6H, $\left.\mathrm{CH}_{3}\right) .{ }^{13} \mathrm{C} \mathrm{NMR}\left(100 \mathrm{MHz}, \mathrm{CDCl}_{3}\right): \delta 151.2,142.4,113.2,88.2$, 76.9, 43.4, 23.1. HRMS calcd for $\mathrm{C}_{11} \mathrm{H}_{18} \mathrm{O}: 166.1358$. Found: 166.1353.

2,6-Dimethyl-4-(vinyloxymethyl)hepta-1,6-diene (15). IR (neat): 3075 (m), 2968 (m), 2924 (s), 2861 (w), 1652 (s), 1608 (s), 1445 (m), 1376 (m), 1325 (m), 1206 (s), 1117 (w), 1073 (w), $998(\mathrm{w}), 966$ (w), 897 (s), 815 (s) $\mathrm{cm}^{-1} .{ }^{1} \mathrm{H}$ NMR (400 MHz, $\mathrm{CDCl}_{3}$ ): $\delta 6.47$ (dd, $J=14.0,6.8$ $\left.\mathrm{Hz}, 1 \mathrm{H}, \mathrm{OCH}=\mathrm{CH}_{2}\right), 4.79\left(\mathrm{~s}, 2 \mathrm{H}, \mathrm{CH}_{3} \mathrm{C}=\mathrm{CH} H^{\prime}\right), 4.70$ (s, 2H, $\left.\mathrm{CH}_{3} \mathrm{C}=\mathrm{C} H \mathrm{H}^{\prime}\right), 4.15$ (dd, J= 14.0, $\left.2.0 \mathrm{~Hz}, 1 \mathrm{H}, \mathrm{OCH}=\mathrm{CH} H^{\prime}\right), 3.95\left(\mathrm{dd}, J=6.8,2.0 \mathrm{~Hz}, 1 \mathrm{H}, \mathrm{OCH}=\mathrm{CH} \mathrm{H}^{\prime}\right), 3.55$ (d, $J=4 \mathrm{~Hz}, 2 \mathrm{H}$, $\left.\mathrm{OCH}_{2}\right), 2.16-1.97\left(\mathrm{~m}, 5 \mathrm{H}, \mathrm{OCH}_{2} \mathrm{CHCH}_{2} \mathrm{CCH}_{3}\right), 1.72\left(\mathrm{~s}, 6 \mathrm{H}, \mathrm{CH}_{3}\right) .{ }^{13} \mathrm{C} \mathrm{NMR}(100 \mathrm{MHz}$, $\left.\mathrm{C}_{6} \mathrm{D}_{6}\right): \delta 152.4,143.8,112.6,86.2,69.7,40.1,34.1,22.3$. HRMS calcd for $\mathrm{C}_{12} \mathrm{H}_{20} \mathrm{O}: 180.1514$. Found: 180.1515 .

(7E,12E)-10-(Vinyloxy)nonadeca-7,12-diene (17). IR (neat): 2955 (m), 2917 (s), 2848 (m), 1634 (m), 1608 (w), 1464 (w), 1432 (w), 1357 (w), 1193 (s), 1174 (m), 966 (s), 815 (w) cm ${ }^{-1}$. ${ }^{1} \mathrm{H}$ NMR (400 MHz, $\left.\mathrm{CDCl}_{3}\right): \delta 6.29\left(\mathrm{dd}, J=14.4,6.0 \mathrm{~Hz}, 1 \mathrm{H}, \mathrm{OCH}=\mathrm{CH}_{2}\right), 5.50-5.30(\mathrm{~m}, 4 \mathrm{H}$, $\mathrm{C} H=\mathrm{C} H), 4.25\left(\mathrm{~d}, J=14.4 \mathrm{~Hz}, 1 \mathrm{H}, \mathrm{OCH}=\mathrm{CH} H^{\prime}\right), 3.95(\mathrm{~d}, J=6.0 \mathrm{~Hz}, 1 \mathrm{H}, \mathrm{OCH}=\mathrm{CHH}), 3.72$ $(\mathrm{tt}, J=6.0,6.0 \mathrm{~Hz}, 1 \mathrm{H}, \mathrm{OCH}), 2.23\left(\mathrm{dd}, J=6.4,6.0 \mathrm{~Hz}, 4 \mathrm{H}, \mathrm{OCHCH}_{2}\right), 2.00-1.95(\mathrm{~m}, 4 \mathrm{H}$, $\left.\mathrm{C}=\mathrm{CHCH}_{2}\right), 1.40-1.20\left(\mathrm{~m}, 16 \mathrm{H},\left(\mathrm{CH}_{2}\right)_{4} \mathrm{CH}_{3}\right), 0.87\left(\mathrm{t}, J=6 \mathrm{~Hz}, 6 \mathrm{H}, \mathrm{CH}_{3}\right) .{ }^{13} \mathrm{C} \mathrm{NMR}(100 \mathrm{MHz}$, $\left.\mathrm{CDCl}_{3}\right): \delta 151.5,134.0,125.3,88.2,79.9,36.9,32.9,31.9,29.6,29.0,22.9,14.3$. HRMS calcd for $\mathrm{C}_{21} \mathrm{H}_{38} \mathrm{O}: 306.2923$. Found: 306.2916.

(7Z,12Z)-10-(Vinyloxy)nonadeca-7,12-diene (19). IR (neat): 2955 (m), 2924 (s), 2855 (m), 1652 (w), 1634 (m), 1602 (w), 1451 (w), 1350 (w), 1325 (w), 1193 (s), 1174 (s), 1060 (w), 815 (m), $721(\mathrm{~m}) \mathrm{cm}^{-1} .{ }^{1} \mathrm{H} \mathrm{NMR}\left(400 \mathrm{MHz}, \mathrm{CDCl}_{3}\right): \delta 6.31\left(\mathrm{dd}, J=14.0,6.4 \mathrm{~Hz}, 1 \mathrm{H}, \mathrm{OCH}=\mathrm{CH}_{2}\right)$, 5.51-5.34 (m, 4H, $\mathrm{CH}=\mathrm{CH}), 4.26\left(\mathrm{dd}, J=14.0,1.2 \mathrm{~Hz}, 1 \mathrm{H}, \mathrm{OCH}=\mathrm{CH} H^{\prime}\right), 3.96(\mathrm{dd}, J=6.4$, $\left.1.2 \mathrm{~Hz}, 1 \mathrm{H}, \mathrm{OCH}=\mathrm{CH} \mathrm{H}^{\prime}\right), 3.75$ (tt, $\left.J=6.0,6.0 \mathrm{~Hz}, 1 \mathrm{H}, \mathrm{OCH}\right), 2.30(\mathrm{dd}, J=6.4,6.0 \mathrm{~Hz}, 4 \mathrm{H}$, $\left.\mathrm{OCHCH}_{2}\right), 2.01\left(\mathrm{dd}, J=14.0,7.2 \mathrm{~Hz}, 4 \mathrm{H}, \mathrm{C}=\mathrm{CHCH}_{2}\right), 1.35-1.20\left(\mathrm{~m}, 16 \mathrm{H},\left(\mathrm{CH}_{2}\right)_{4} \mathrm{CH}_{3}\right), 0.87$ (t, $\left.J=7.2 \mathrm{~Hz}, 6 \mathrm{H}, \mathrm{CH}_{3}\right) .{ }^{13} \mathrm{C} \mathrm{NMR}\left(100 \mathrm{MHz}, \mathrm{CDCl}_{3}\right): \delta 151.5,132.9,124.7,88.2,80.1,32.0$, 31.8, 29.7, 29.2, 27.7, 22.8, 14.3. HRMS calcd for $\mathrm{C}_{21} \mathrm{H}_{38} \mathrm{O}: 306.2923$. Found: 306.2919.

(2,6-Dimethyl-4-(vinyloxymethyl)hepta-1,6-dien-4-yl)cyclohexane (21). IR (neat): 2924 (s), 2855 (m), 1646 (w), 1602 (m), 1451 (w), 1369 (w), 1313 (w), 1199 (s), 885 (m), 809 (w) cm ${ }^{-1}$. ${ }^{1} \mathrm{H}$ NMR $\left(400 \mathrm{MHz}, \mathrm{CDCl}_{3}\right): \delta 6.47\left(\mathrm{dd}, J=14.4,6.9 \mathrm{~Hz}, 1 \mathrm{H}, \mathrm{OCH}=\mathrm{CH}_{2}\right), 4.84(\mathrm{~s}, 2 \mathrm{H}$, $\left.\mathrm{CH}_{3} \mathrm{C}=\mathrm{CH} H^{\prime}\right), 4.68\left(\mathrm{~s}, 2 \mathrm{H}, \mathrm{CH}_{3} \mathrm{C}=\mathrm{CHH}^{\prime}\right), 4.09\left(\mathrm{dd}, J=14.4,1.8 \mathrm{~Hz}, 1 \mathrm{H}, \mathrm{OCH}=\mathrm{CH} H^{\prime}\right), 3.95$ 
$\left(\mathrm{dd}, J=6.9,1.8 \mathrm{~Hz}, 1 \mathrm{H}, \mathrm{OCH}=\mathrm{CH} \mathrm{H}^{\prime}\right), 3.53\left(\mathrm{~s}, 2 \mathrm{H}, \mathrm{OCH}_{2}\right), 2.17(\mathrm{~d}, J=13.7 \mathrm{~Hz}, 2 \mathrm{H}$, $\left.\mathrm{CH}^{\prime} \mathrm{CCH}_{3}\right), 2.12\left(\mathrm{~d}, J=13.2 \mathrm{~Hz}, 2 \mathrm{H}, \mathrm{CH}^{\prime} \mathrm{CCH}_{3}\right), 1.74\left(\mathrm{~s}, 6 \mathrm{H}, \mathrm{CH}_{2} \mathrm{CCH}_{3}\right), 1.78-1.46$ (m, $5 \mathrm{H}, \mathrm{Cy}-H), 1.26-1.03(\mathrm{~m}, 6 \mathrm{H}, \mathrm{Cy}-H) .{ }^{13} \mathrm{C} \mathrm{NMR}\left(100 \mathrm{MHz}, \mathrm{CDCl}_{3}\right): \delta 151.6,143.7,114.9$, 86.1, 71.9, 43.1, 43.0, 41.6, 27.6, 27.4, 27.0, 25.6. HRMS calcd for $\mathrm{C}_{18} \mathrm{H}_{30} \mathrm{O}: 262.2297$. Found: 262.2295 .

(2,6-Dimethyl-4-(vinyloxymethyl)hepta-1,6-dien-4-yl)benzene (23a). IR (neat): 2943 (m), 1640 (m), 1615 (m), 1451 (m), 1376 (w), 1319 (w), 1199 (s), 1011 (w), 897 (s), 815 (w), 765 (w), $702(\mathrm{~m}) \mathrm{cm}^{-1} .{ }^{1} \mathrm{H}$ NMR (400 MHz, $\left.\mathrm{CDCl}_{3}\right): \delta 7.30-7.15(\mathrm{~m}, 5 \mathrm{H}, \mathrm{Ar}-H), 6.57$ (dd, $J=14.4$, $\left.6.8 \mathrm{~Hz}, 1 \mathrm{H}, \mathrm{OCH}=\mathrm{CH}_{2}\right), 4.72\left(\mathrm{~s}, 2 \mathrm{H}, \mathrm{CH}_{3} \mathrm{C}=\mathrm{CH} H^{\prime}\right), 4.50\left(\mathrm{~s}, 2 \mathrm{H}, \mathrm{CH}_{3} \mathrm{C}=\mathrm{C} H \mathrm{H}^{\prime}\right), 4.27(\mathrm{~d}, J=$ $\left.14.4 \mathrm{~Hz}, 1 \mathrm{H}, \mathrm{OCH}=\mathrm{CH} H^{\prime}\right), 4.03\left(\mathrm{~d}, J=6.8 \mathrm{~Hz}, 1 \mathrm{H}, \mathrm{OCH}=\mathrm{CH} \mathrm{H}^{\prime}\right), 3.98\left(\mathrm{~s}, 2 \mathrm{H}, \mathrm{OCH}_{2}\right), 2.62(\mathrm{~d}$, $\left.J=13.6 \mathrm{~Hz}, 2 \mathrm{H}, \mathrm{CH} H^{\prime} \mathrm{CCH}_{3}\right), 2.44\left(\mathrm{~d}, J=13.6 \mathrm{~Hz}, 2 \mathrm{H}, \mathrm{C} H \mathrm{H}^{\prime} \mathrm{CCH}_{3}\right), 1.24\left(\mathrm{~s}, 6 \mathrm{H}, \mathrm{CH}_{3}\right) .{ }^{13} \mathrm{C}$ NMR (100 MHz, $\left.\mathrm{CDCl}_{3}\right): \delta 151.8,144.6,142.5,128.2,126.9,126.3,115.1,86.5,68.2,46.5$, 44.4, 24.8. HRMS calcd for $\mathrm{C}_{18} \mathrm{H}_{24} \mathrm{O}: 256.1827$. Found: 256.1820.

1-(2,6-Dimethyl-4-(vinyloxymethyl)hepta-1,6-dien-4-yl)-4-methoxybenzene (23b). IR (neat): 3075 (m), 2943 (s), 2836 (m), 1646 (s), 1615 (s), 1514 (s), 1457 (s), 1376 (m), 1319 (m), 1294 (m), 1250 (s), 1199 (s), 1036 (s), 1011 (m), 904 (s), 828 (s) cm ${ }^{-1}$. ${ }^{1}$ H NMR (400 $\mathrm{MHz}_{\mathrm{CDCl}}$ ): $\delta 7.15(\mathrm{~d}, J=9.2 \mathrm{~Hz}, 2 \mathrm{H}, \mathrm{Ar}-\mathrm{H}), 6.82(\mathrm{~d}, J=9.2 \mathrm{~Hz}, 2 \mathrm{H}, \mathrm{Ar}-\mathrm{H}), 6.56(\mathrm{dd}, J=$ 14.6, $\left.6.6 \mathrm{~Hz}, 1 \mathrm{H}, \mathrm{OCH}=\mathrm{CH}_{2}\right), 4.72\left(\mathrm{~s}, 2 \mathrm{H}, \mathrm{CH}_{3} \mathrm{C}=\mathrm{CH} H^{\prime}\right), 4.49\left(\mathrm{~s}, 2 \mathrm{H}, \mathrm{CH}_{3} \mathrm{C}=\mathrm{CH} \mathrm{H}^{\prime}\right), 4.26(\mathrm{~d}$, $\left.J=14.4 \mathrm{~Hz}, 1 \mathrm{H}, \mathrm{OCH}=\mathrm{CH} H^{\prime}\right), 4.02\left(\mathrm{~d}, J=6.8 \mathrm{~Hz}, 1 \mathrm{H}, \mathrm{OCH}=\mathrm{CHH}^{\prime}\right), 3.94\left(\mathrm{~s}, 2 \mathrm{H}, \mathrm{OCH}_{2}\right)$, $3.78\left(\mathrm{~s}, 3 \mathrm{H}, \mathrm{OCH}_{3}\right), 2.59\left(\mathrm{~d}, J=13.2 \mathrm{~Hz}, 2 \mathrm{H}, \mathrm{CH}^{\prime} \mathrm{CCH}_{3}\right), 2.40(\mathrm{~d}, J=13.2 \mathrm{~Hz}, 2 \mathrm{H}$, $\left.\mathrm{CHH}^{\prime} \mathrm{CCH}_{3}\right), 1.26\left(\mathrm{~s}, 6 \mathrm{H}, \mathrm{CH}_{3}\right) .{ }^{13} \mathrm{C} \mathrm{NMR}\left(100 \mathrm{MHz}, \mathrm{CDCl}_{3}\right): \delta 157.9,151.8,142.7,136.5$, 127.8, 115.0, 113.5, 86.5, 68.4, 55.3, 46.5, 43.8, 24.9. HRMS calcd for $\mathrm{C}_{18} \mathrm{H}_{26} \mathrm{O}_{2}$ : 286.1933. Found: 286.1931.

1-Bromo-4-(2,6-dimethyl-4-(vinyloxymethyl)hepta-1,6-dien-4-yl)benzene (23c). $\mathrm{mp}=52-$ $53{ }^{\circ} \mathrm{C}$ (hexane). IR (neat): 3069 (w), 2943 (m), 1640 (s), 1615 (s), 1489 (s), 1451 (m), 1325 (w), 1199 (s), 1086 (m), 1010 (s), 897 (s), 822 (s) cm ${ }^{-1} .{ }^{1} \mathrm{H}$ NMR (400 MHz, CDCl $): \delta 7.40$ (d, $J=8.8 \mathrm{~Hz}, 2 \mathrm{H}, \operatorname{Ar}-H), 7.13(\mathrm{~d}, J=8.8 \mathrm{~Hz}, 2 \mathrm{H}, \mathrm{Ar}-H), 6.54(\mathrm{dd}, J=14.4,6.8 \mathrm{~Hz}, 1 \mathrm{H}$, $\left.\mathrm{OCH}=\mathrm{CH}_{2}\right), 4.74\left(\mathrm{~s}, 2 \mathrm{H}, \mathrm{CH}_{3} \mathrm{C}=\mathrm{CH} H^{\prime}\right), 4.49\left(\mathrm{~s}, 2 \mathrm{H}, \mathrm{CH}_{3} \mathrm{C}=\mathrm{CH} \mathrm{H}^{\prime}\right), 4.25(\mathrm{~d}, J=14.4 \mathrm{~Hz}, 1 \mathrm{H}$, $\left.\mathrm{OCH}=\mathrm{CH} H^{\prime}\right), 4.04\left(\mathrm{~d}, J=6.8 \mathrm{~Hz}, 1 \mathrm{H}, \mathrm{OCH}=\mathrm{C} H \mathrm{H}^{\prime}\right), 3.92\left(\mathrm{~s}, 2 \mathrm{H}, \mathrm{OCH}_{2}\right), 2.59(\mathrm{~d}, J=13.2 \mathrm{~Hz}$, $\left.2 \mathrm{H}, \mathrm{CH}^{\prime} \mathrm{CCH}_{3}\right), 2.40\left(\mathrm{~d}, J=13.2 \mathrm{~Hz}, 2 \mathrm{H}, \mathrm{CHH}^{\prime} \mathrm{CCH}_{3}\right), 1.28\left(\mathrm{~s}, 6 \mathrm{H}, \mathrm{CH}_{3}\right) .{ }^{13} \mathrm{C}$ NMR $(100$ $\left.\mathrm{MHz}, \mathrm{CDCl}_{3}\right): \delta 151.6,143.8,142.0,131.3,128.8,120.2,115.4,86.8,68.1,46.2,44.4,25.0$. HRMS calcd for $\mathrm{C}_{18} \mathrm{H}_{23} \mathrm{BrO}$ : 334.0932. Found: 334.0936. 
Methyl 4-methyl-2-(2-methylallyl)-2-(vinyloxymethyl)pent-4-enoate (25). IR (neat): 3075 (w), 2949 (m), 1734 (s), 1646 (w), 1615 (m), 1457 (w), 1369 (w), 1325 (w), 1199 (s), 1086 (w), $1061(\mathrm{w}), 1011(\mathrm{w}), 897(\mathrm{~m}), 809(\mathrm{w}) \mathrm{cm}^{-1} .{ }^{1} \mathrm{H}$ NMR (400 MHz, $\left.\mathrm{C}_{6} \mathrm{D}_{6}\right): \delta 6.33$ (dd, $J=14.8$, $\left.6.8 \mathrm{~Hz}, 1 \mathrm{H}, \mathrm{OCH}=\mathrm{CH}_{2}\right), 4.84-4.81\left(\mathrm{~m}, 2 \mathrm{H}, \mathrm{CH}_{3} \mathrm{C}=\mathrm{CH} H^{\prime}\right), 4.81-4.79\left(\mathrm{~m}, 2 \mathrm{H}, \mathrm{CH}_{3} \mathrm{C}=\mathrm{CHH}^{\prime}\right)$, $4.10\left(\mathrm{dd}, J=14.8,2.0 \mathrm{~Hz}, 1 \mathrm{H}, \mathrm{OCH}=\mathrm{CH} H^{\prime}\right), 3.93\left(\mathrm{~s}, 2 \mathrm{H}, \mathrm{OCH}_{2}\right), 3.87(\mathrm{dd}, J=6.8,2.0 \mathrm{~Hz}$, $\left.1 \mathrm{H}, \mathrm{OCH}=\mathrm{CH} \mathrm{H}^{\prime}\right), 3.23\left(\mathrm{~s}, 3 \mathrm{H}, \mathrm{COOCH}_{3}\right), 2.45\left(\mathrm{~s}, 4 \mathrm{H}, \mathrm{CH}_{2} \mathrm{C}=\mathrm{CH}_{2}\right), 1.58\left(\mathrm{~s}, 6 \mathrm{H}, \mathrm{CH}_{3}\right) .{ }^{13} \mathrm{C}$ NMR $\left(100 \mathrm{MHz}, \mathrm{C}_{6} \mathrm{D}_{6}\right): \delta 174.6,151.3,141.4,115.3,86.7,65.7,50.8,49.8,43.2,23.6$. HRMS calcd for $\mathrm{C}_{14} \mathrm{H}_{22} \mathrm{O}_{3}: 238.1569$. Found: 238.1574 .

Benzyl 2,6-dimethyl-4-(vinyloxymethyl)hepta-1,6-dien-4-ylcarbamate (27). IR (neat): 3345 (br), 3075 (w), 2943 (m), 1728 (s), 1709 (s), 1646 (w), 1621 (m), 1508 (s), 1457 (w), 1375 (w), 1319 (w), 1231 (s), 1199 (s), 1055 (m), 904 (s), 690 (m) cm ${ }^{-1} .{ }^{1} \mathrm{H}$ NMR (400 MHz, $\mathrm{C}_{6} \mathrm{D}_{6}$ ): $\delta$ 7.21-6.99 (m, 5H, Ar-H), $6.21\left(\mathrm{dd}, J=14.8,6.8 \mathrm{~Hz}, 1 \mathrm{H}, \mathrm{OCH}=\mathrm{CH}_{2}\right), 4.99\left(\mathrm{~s}, 2 \mathrm{H}, \mathrm{PhCH}_{2}\right)$, $4.81\left(\mathrm{~s}, 2 \mathrm{H}, \mathrm{CH}_{3} \mathrm{C}=\mathrm{CH} H^{\prime}\right), 4.70\left(\mathrm{~s}, 2 \mathrm{H}, \mathrm{CH}_{3} \mathrm{C}=\mathrm{C} H \mathrm{H}^{\prime}\right), 4.67$ (br s, $\left.1 \mathrm{H}, \mathrm{NH}\right), 4.06(\mathrm{dd}, J=14.8$, $\left.2.0 \mathrm{~Hz}, 1 \mathrm{H}, \mathrm{OCH}=\mathrm{CH} H^{\prime}\right), 3.85$ (dd, $\left.J=6.8,2.0 \mathrm{~Hz}, 1 \mathrm{H}, \mathrm{OCH}=\mathrm{CH} \mathrm{H}^{\prime}\right), 3.57\left(\mathrm{~s}, 2 \mathrm{H}, \mathrm{OCH}_{2}\right)$, $2.72\left(\mathrm{~d}, J=14.0 \mathrm{~Hz}, 2 \mathrm{H}, \mathrm{CH}^{\prime} \mathrm{CCH}_{3}\right), 2.58\left(\mathrm{~d}, J=14.0 \mathrm{~Hz}, 2 \mathrm{H}, \mathrm{CHH}^{\prime} \mathrm{CCH}_{3}\right), 1.64(\mathrm{~s}, 6 \mathrm{H}$, $\left.\mathrm{CH}_{3}\right) .{ }^{13} \mathrm{C} \mathrm{NMR}\left(100 \mathrm{MHz}, \mathrm{CDCl}_{3}\right): \delta 151.1,141.8,136.8,128.7,128.4,128.3,116.1,87.4$, 69.5, 66.5, 57.3, 41.6, 24.6. HRMS calcd for $\mathrm{C}_{20} \mathrm{H}_{27} \mathrm{NO}_{3}$ : 329.1991. Found: 329.1983.

\section{Representative procedure for Mo-catalyzed asymmetric ring-closing olefin metathesis of}

enol ethers: ${ }^{7}$ A one-dram vial containing a stir bar was charged with $23 \mathbf{a}(12.8 \mathrm{mg}, 50.0 \mu \mathrm{mol})$ in a $\mathrm{N}_{2}$-filled glove box. A solution of complex $(R)-4 \mathbf{a}(8.7 \mathrm{mg}, 7.5 \mathrm{mmol})$ in benzene $(0.5 \mathrm{~mL})$ was added in one portion. The vial was loosely covered with a Teflon-lined cap and the reaction was allowed to stir at $22{ }^{\circ} \mathrm{C}$ for $20 \mathrm{~h}$. The reaction vessel was then removed from the glove box and quenched by exposure to air. The solution was concentrated by purging with a stream of $\mathrm{N}_{2}$ to give a brown residue. Purification by silica gel column chromatography with 49:1 pentane: $\mathrm{Et}_{2} \mathrm{O}$ as the eluent afforded $\mathbf{2 4 a}(11.0 \mathrm{mg}, 48.0 \mu \mathrm{mol}, 96 \%$ yield).

(R)-4-Methyl-2-(2-methylallyl)-2,3-dihydrofuran (13). $[\alpha]_{D}^{20}+44.3\left(c=0.857, \mathrm{Et}_{2} \mathrm{O}\right)$. IR (neat): 3069 (m), 2918 (s), 2855 (s), 1678 (s), 1652 (m), 1451 (s), 1376 (m), 1300 (w), 1262 (w), 1099 (s), 1010 (w), 891 (s), 834 (m) cm ${ }^{-1} .{ }^{1} \mathrm{H}$ NMR (400 MHz, $\left.\mathrm{C}_{6} \mathrm{D}_{6}\right): \delta 5.98$ (d, $J=1.6$ $\left.\mathrm{Hz}, 1 \mathrm{H}, \mathrm{OCH}=\mathrm{CCH}_{3}\right), 4.81\left(\mathrm{~s}, 1 \mathrm{H}, \mathrm{C}=\mathrm{CH}_{2}\right), 4.74\left(\mathrm{~s}, 1 \mathrm{H}, \mathrm{C}=\mathrm{CH}_{2}\right), 4.69-4.65(\mathrm{~m}, 1 \mathrm{H}$,

7) Complexes 3a-3b were of the $S$ configuration and complex $4 \mathbf{a}$ of the $R$ configuration. To facilitate comparison between biphen- and binol-based catalysts, all Mo-based complexes in Scheme 1 of the article were depicted as $S$ isomers. The enantiomer of the products illustrated is what would be obtained with the antipode of catalyst illustrated in Scheme 1 (see text). 
$\left.\mathrm{OCHCH}_{2}\right), 2.59\left(\mathrm{dd}, J=14.4,9.6 \mathrm{~Hz}, 1 \mathrm{H}, \mathrm{H}_{2} \mathrm{C}=\mathrm{CCH}_{2}\right), 2.43(\mathrm{dd}, J=14.4,7.2 \mathrm{~Hz}, 1 \mathrm{H}$, $\left.\mathrm{H}_{2} \mathrm{C}=\mathrm{CCH}_{2}\right), 2.24-2.17\left(\mathrm{~m}, 2 \mathrm{H}, \mathrm{OCH}=\mathrm{CCH}_{2}\right), 1.76\left(\mathrm{~s}, 3 \mathrm{H}, \mathrm{CH}_{3}\right), 1.62\left(\mathrm{~s}, 3 \mathrm{H}, \mathrm{CH}_{3}\right) .{ }^{13} \mathrm{C} \mathrm{NMR}$ $\left(100 \mathrm{MHz}, \mathrm{C}_{6} \mathrm{D}_{6}\right): \delta 142.5,140.0,112.7,108.4,80.1,44.7,39.8,22.9,11.3$. HRMS calcd for $\left[\mathrm{C}_{9} \mathrm{H}_{14} \mathrm{O}\right]+\mathrm{H}$ : 139.1123. Found 139.1125 . The optical purity of $\mathbf{1 3}(90 \%$ ee) was determined by chiral GC analysis (CDGTA column, $15 \mathrm{psi}, 40{ }^{\circ} \mathrm{C}$; $\mathrm{t}_{\mathrm{r}}$ (major enantiomer) $=31.8 \mathrm{~min}, \mathrm{t}_{\mathrm{r}}$ (minor enantiomer $)=36.2 \mathrm{~min})$. Chromatograms are illustrated below:
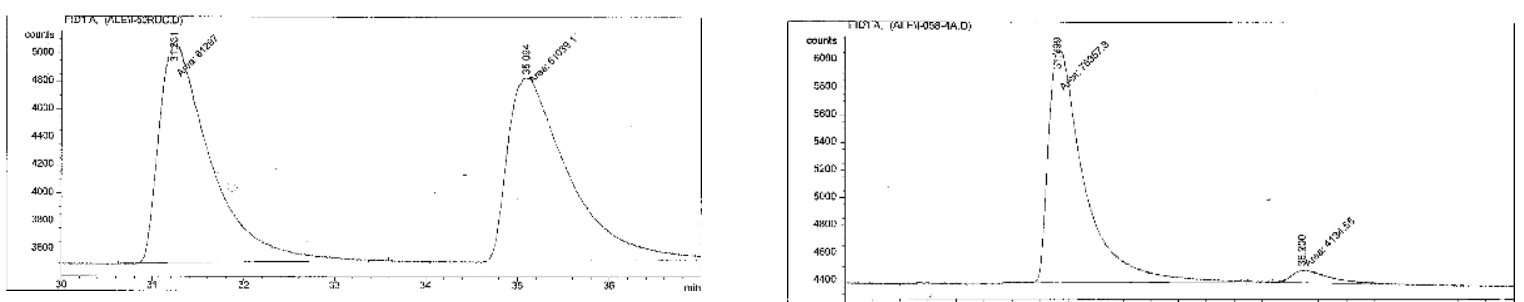

(R)-5-Methyl-2-(3-methylbut-3-enyl)-3,4-dihydro-2H-pyran (14). $[\alpha]_{D}^{20}+66.7(c=0.466$, $\mathrm{CHCl}_{3}$ ). IR (neat): 2917 (s), 2854 (m), 1671 (m), 1652 (w), 1445 (m), 1167 (s), 1148 (s), 891 (w) $\mathrm{cm}^{-1} .{ }^{1} \mathrm{H}$ NMR $\left(400 \mathrm{MHz}, \mathrm{C}_{6} \mathrm{D}_{6}\right): \delta 6.27\left(\mathrm{~d}, J=1.1 \mathrm{~Hz}, 1 \mathrm{H}, \mathrm{OCH}=\mathrm{CCH}_{3}\right), 4.74(\mathrm{~s}, 2 \mathrm{H}$, $\left.\mathrm{CH}_{3} \mathrm{C}=\mathrm{CH}_{2}\right), 3.55-3.49\left(\mathrm{~m}, 1 \mathrm{H}, \mathrm{CH}_{2} \mathrm{CHO}\right), 2.17$ (ddd, $J=15.3,10.4,5.4 \mathrm{~Hz}, 1 \mathrm{H}, \mathrm{H}_{2} \mathrm{C}=\mathrm{CCH}_{2}$ ), $2.00\left(\mathrm{ddd}, J=15.3,10.1,5.9 \mathrm{~Hz}, 1 \mathrm{H}, \mathrm{H}_{2} \mathrm{C}=\mathrm{CCH}_{2}\right), 1.85-1.75(\mathrm{~m}, 1 \mathrm{H}, \mathrm{CH}), 1.75-1.65(\mathrm{~m}, 1 \mathrm{H}$, $\left.\mathrm{CH}_{2}\right), 1.63-1.53\left(\mathrm{~m}, 1 \mathrm{H}, \mathrm{CH}_{2}\right), 1.58\left(\mathrm{~s}, 3 \mathrm{H}, \mathrm{CH}_{3} \mathrm{C}=\mathrm{CH}_{2}\right), 1.41\left(\mathrm{~d}, J=1.1 \mathrm{~Hz}, 3 \mathrm{H}, \mathrm{HC}=\mathrm{CCH}_{3}\right)$, 1.50-1.33 (m, 3H, $\left.\mathrm{CH}_{2}\right) .{ }^{13} \mathrm{C} \mathrm{NMR}\left(100 \mathrm{MHz}, \mathrm{C}_{6} \mathrm{D}_{6}\right): \delta 145.4,139.2,110.2,107.4,73.9,33.7$, 33.5, 28.0, 25.6, 22.4, 18.3. HRMS calcd for $\mathrm{C}_{11} \mathrm{H}_{18} \mathrm{O}$ : 166.1358. Found: 166.1354. The optical purity of 14 (83\% ee) was determined by chiral GC analysis $\left(\beta\right.$-Dex column, $15 \mathrm{psi}, 60{ }^{\circ} \mathrm{C}$; $\mathrm{t}_{\mathrm{r}}$ $($ minor enantiomer $)=289.0 \mathrm{~min}, \mathrm{t}_{\mathrm{r}}($ major enantiomer $\left.)=292.4 \mathrm{~min}\right)$. Chromatograms are illustrated below:
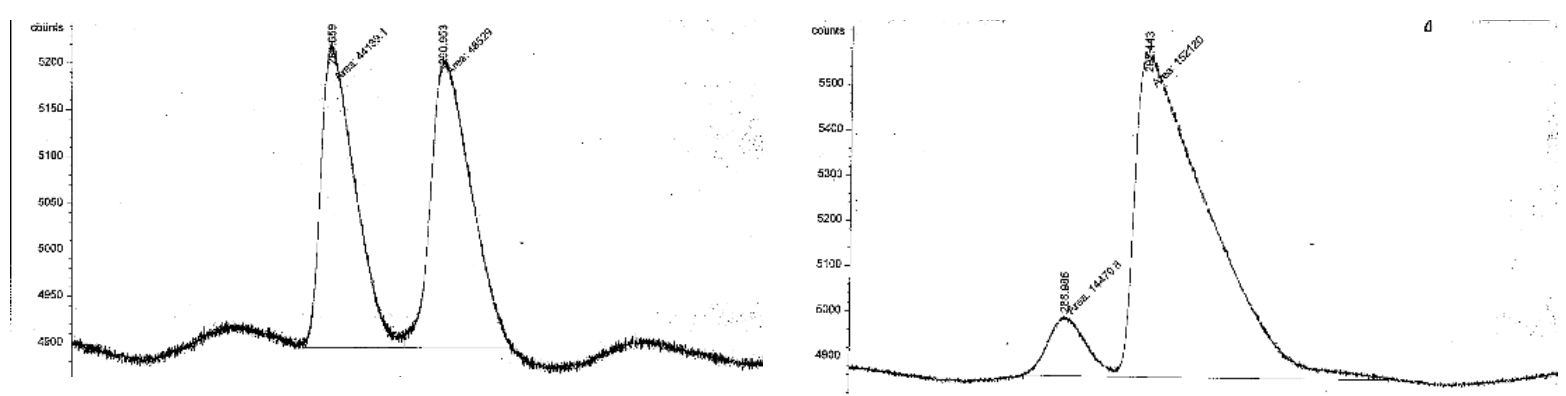
(R)-5-Methyl-3-(2-methylallyl)-3,4-dihydro-2H-pyran (16). $[\alpha]_{D}^{20}-3.8\left(c=0.200, \mathrm{CHCl}_{3}\right)$. IR (neat): 2962 (w), 2924 (s), 2848 (m), 1639 (w), 1464 (m), 1376 (w), 1255 (w) cm ${ }^{-1} .{ }^{1} \mathrm{H}$ NMR (400 MHz, $\left.\mathrm{CDCl}_{3}\right): \delta 6.20\left(\mathrm{dd}, J=3.3,1.7 \mathrm{~Hz}, 1 \mathrm{H}, \mathrm{OCH}=\mathrm{CCH}_{3}\right), 4.79-4.78(\mathrm{~m}, 1 \mathrm{H}$, $\left.\mathrm{CH}_{3} \mathrm{C}=\mathrm{CH}_{2}\right), 4.71-4.69\left(\mathrm{~m}, 1 \mathrm{H}, \mathrm{CH}_{3} \mathrm{C}=\mathrm{CH}_{2}\right), 3.91\left(\mathrm{ddd}, J=10.3,3.1,1.7 \mathrm{~Hz}, 1 \mathrm{H}, \mathrm{OCH}_{2}\right), 3.41$ $\left(\mathrm{dd}, J=10.3,8.8 \mathrm{~Hz}, 1 \mathrm{H}, \mathrm{OCH}_{2}\right), 2.03-1.90\left(\mathrm{~m}, 4 \mathrm{H}, \mathrm{CH}_{2}\right), 1.73\left(\mathrm{~s}, 3 \mathrm{H}, \mathrm{CH}_{3}\right), 1.62(\mathrm{dd}, J=$ 16.7, $\left.8.8 \mathrm{~Hz}, 1 \mathrm{H}, \mathrm{OCH}_{2} \mathrm{CH}\right), 1.54\left(\mathrm{dd}, J=2.2,1.1 \mathrm{~Hz}, 3 \mathrm{H}, \mathrm{CH}_{3}\right) .{ }^{13} \mathrm{C} \mathrm{NMR}(100 \mathrm{MHz}$, $\left.\mathrm{CDCl}_{3}\right): \delta 143.2,138.3,112.2,108.1,69.3,40.9,31.9,30.3,22.4,18.6$. HRMS calcd for $\mathrm{C}_{10} \mathrm{H}_{16} \mathrm{O}:$ 152.1201. Found: 152.1199. The optical purity of $\mathbf{1 6}(90 \%$ ee) was determined by chiral GC analysis (CDGTA column, $20 \mathrm{psi}, 45^{\circ} \mathrm{C}$; $\mathrm{t}_{\mathrm{r}}$ (major enantiomer) $=53.0 \mathrm{~min}, \mathrm{t}_{\mathrm{r}}($ minor enantiomer $)=56.7 \mathrm{~min})$. Chromatograms are illustrated below:
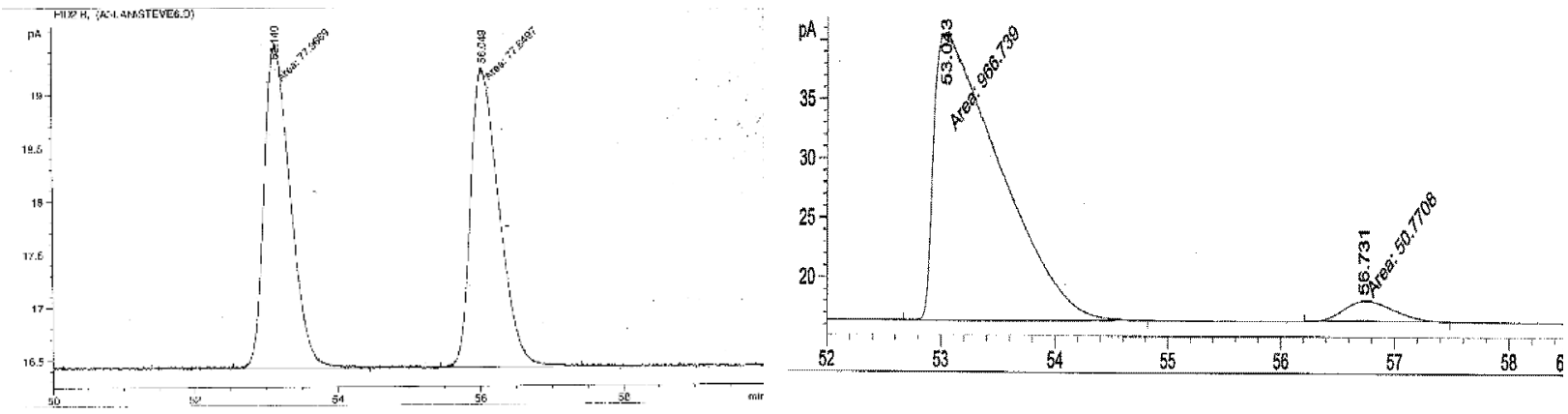

$(\boldsymbol{S}, \boldsymbol{E})-2-\left(\right.$ Non-2-enyl)-2,3-dihydrofuran (18). $[\alpha]_{D}^{20}+14.5\left(c=0.200, \mathrm{CHCl}_{3}\right)$. IR (neat): 2924 (s), 2848 (m), 1615 (m), 1457 (w), 1180 (w), 1143 (m), 1055 (m), 966 (w), 702 (w) cm ${ }^{-1} .{ }^{1} \mathrm{H}$ NMR (400 MHz, $\left.\mathrm{C}_{6} \mathrm{D}_{6}\right): \delta 6.16(\mathrm{~d}, J=1.6 \mathrm{~Hz}, 1 \mathrm{H}, \mathrm{OCH}=\mathrm{CH}), 5.44-5.38(\mathrm{~m}, 2 \mathrm{H}$, $\left.\mathrm{C}_{6} \mathrm{H}_{13} \mathrm{CH}=\mathrm{CH}\right), 4.602-4.59(\mathrm{~m}, 1 \mathrm{H}, \mathrm{OCH}=\mathrm{CH}), 4.43-4.40\left(\mathrm{~m}, 1 \mathrm{H}, \mathrm{CH}_{2} \mathrm{CHO}\right), 2.40-1.85$ (m, $\left.6 \mathrm{H}, \mathrm{CH}_{2}\right), 1.30-1.10\left(\mathrm{~m}, 8 \mathrm{H}, \mathrm{CH}_{2}\right), 0.82\left(\mathrm{t}, J=16.8 \mathrm{~Hz}, 3 \mathrm{H}, \mathrm{CH}_{3}\right) .{ }^{13} \mathrm{C} \mathrm{NMR}\left(100 \mathrm{MHz}, \mathrm{C}_{6} \mathrm{D}_{6}\right)$ : $\delta 145.5,133.5,125.5,98.5,80.9,39.3,34.1,32.9,31.9,29.7,29.0,22.9,14.2$. HRMS calcd for $\mathrm{C}_{13} \mathrm{H}_{22} \mathrm{O}$ : 194.1671. Found: 194.1673 . The optical purity of $\mathbf{1 8}$ (41\% ee) was determined by chiral GC analysis (CDGTA column, $15 \mathrm{psi}, 60{ }^{\circ} \mathrm{C}$; $\mathrm{t}_{\mathrm{r}}$ (major enantiomer) $=240.2 \mathrm{~min}, \mathrm{t}_{\mathrm{r}}$ $($ minor enantiomer $)=246.2 \mathrm{~min})$. Chromatograms are illustrated below: 

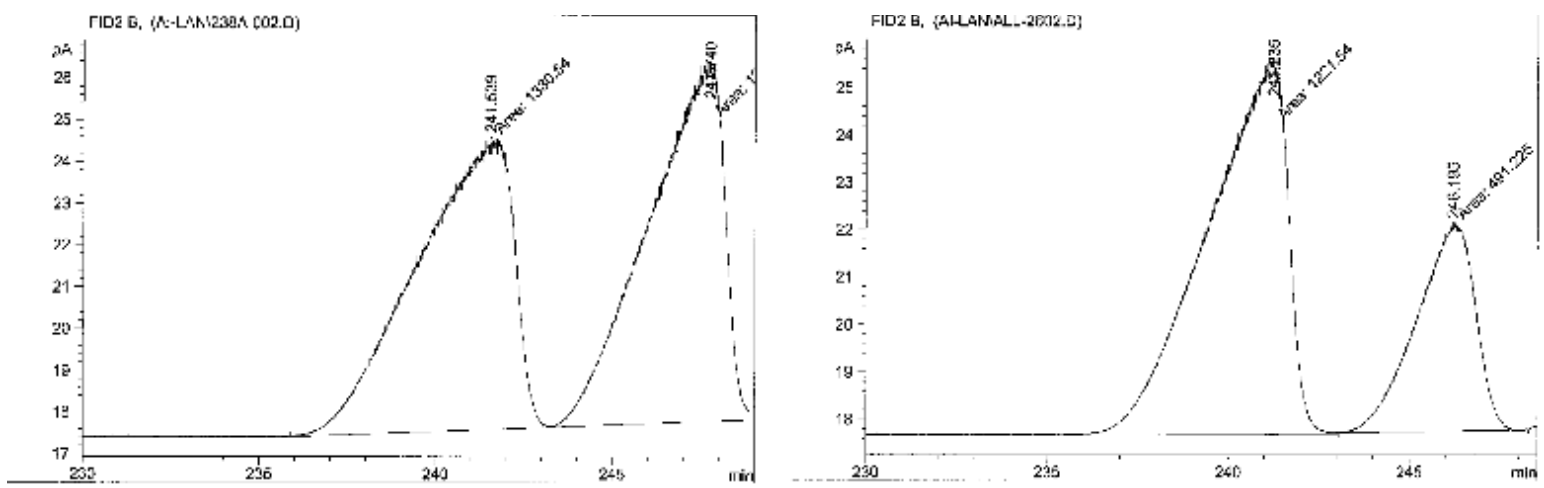

(S,Z)-2-(Non-2-enyl)-2,3-dihydrofuran (20). $[\alpha]_{D}^{20}-42.4\left(c=0.200, \mathrm{CHCl}_{3}\right)$. IR (neat): 2926 (s), 2852 (m), 1618 (w), 1558 (w), 1540 (w), 1140 (w), 1056 (m) cm ${ }^{-1} .{ }^{1} \mathrm{H}$ NMR (400 MHz, $\left.\mathrm{CDCl}_{3}\right): \delta 6.25(\mathrm{dd}, J=4.8,2.4 \mathrm{~Hz}, 1 \mathrm{H}, \mathrm{OCH}=\mathrm{CH}), 5.53-5.48(\mathrm{~m}, 1 \mathrm{H}, \mathrm{CH}=\mathrm{CH}), 5.41-5.36(\mathrm{~m}$, $1 \mathrm{H}, \mathrm{CH}=\mathrm{CH}), 4.83-4.81(\mathrm{~m}, 1 \mathrm{H}, \mathrm{OCH}=\mathrm{CH}), 4.58-4.50\left(\mathrm{~m}, 1 \mathrm{H}, \mathrm{CH}_{2} \mathrm{CHO}\right), 2.68-2.60(\mathrm{~m}, 1 \mathrm{H}$, $\left.\mathrm{CH}_{2}\right), 2.45-2.20\left(\mathrm{~m}, 3 \mathrm{H}, \mathrm{CH}_{2}\right), 2.01-1.90\left(\mathrm{~m}, 2 \mathrm{H}, \mathrm{CH}_{2}\right), 1.33-1.24\left(\mathrm{~m}, 8 \mathrm{H}, \mathrm{CH}_{2}\right), 0.87(\mathrm{t}, J=13.6$ $\left.\mathrm{Hz}, 3 \mathrm{H}, \mathrm{CH}_{3}\right) .{ }^{13} \mathrm{C} \mathrm{NMR}\left(100 \mathrm{MHz}, \mathrm{CDCl}_{3}\right): \delta 145.1,134.0,125.1,99.1,81.2,39.2,34.1,32.9$, 31.9, 29.6, 29.0, 22.8, 14.3. HRMS calcd for $\mathrm{C}_{13} \mathrm{H}_{22} \mathrm{O}$ : 194.1671. Found: 194.1666. The optical purity of 20 (62\% ee) was determined by chiral GC analysis (CDGTA column, 15 psi, $60{ }^{\circ} \mathrm{C}$; $\mathrm{t}_{\mathrm{r}}$ $($ minor enantiomer $)=240.7 \mathrm{~min}, \mathrm{t}_{\mathrm{r}}($ major enantiomer $\left.)=246.4 \mathrm{~min}\right)$. Chromatograms are illustrated below:
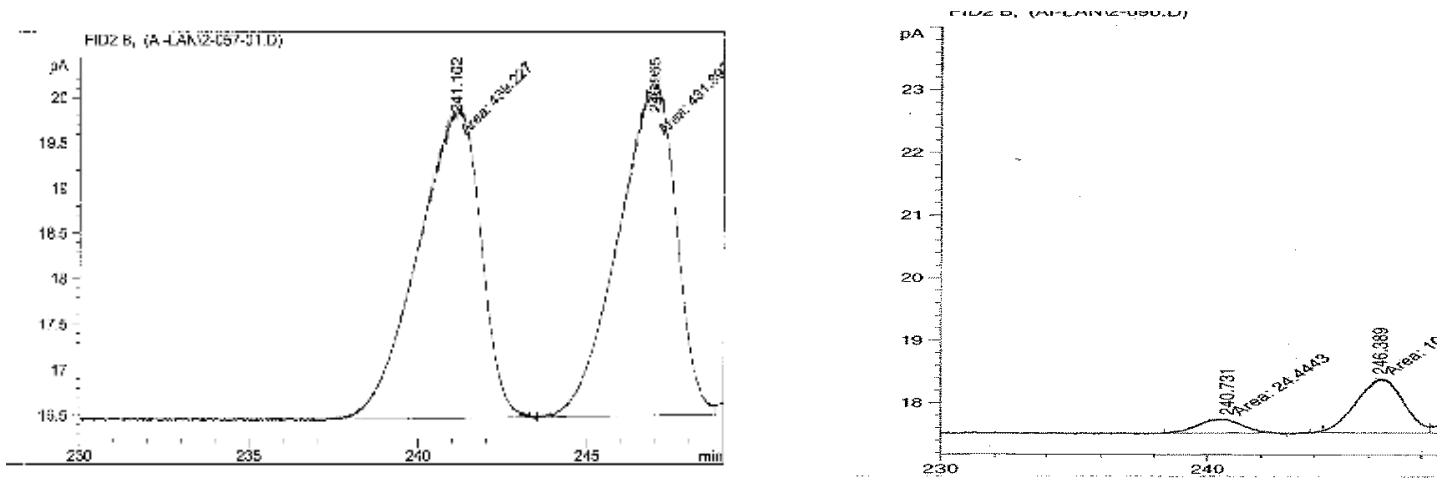

(R)-3-Cyclohexyl-5-methyl-3-(2-methylallyl)-3,4-dihydro-2H-pyran (22). $[\alpha]_{D}^{20} \quad 0 \quad(c=$ 0.353, $\mathrm{CHCl}_{3}$ ). IR (neat): 2924 (s), 2848 (m), 1659 (w), 1652 (w), 1508 (m), 1451 (s), 1149 (s),

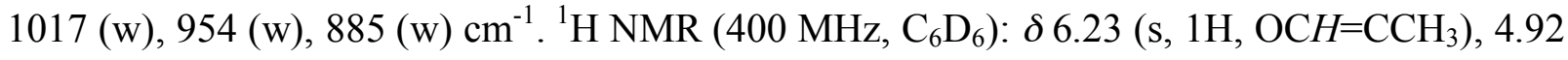
$\left(\mathrm{s}, 1 \mathrm{H}, \mathrm{CH}_{3} \mathrm{C}=\mathrm{CH} H^{\prime}\right), 4.81\left(\mathrm{~s}, 1 \mathrm{H}, \mathrm{CH}_{3} \mathrm{C}=\mathrm{CHH}^{\prime}\right), 3.65\left(\mathrm{~d}, J=10.8 \mathrm{~Hz}, 1 \mathrm{H}, \mathrm{CH}_{2} \mathrm{O}\right), 3.57(\mathrm{~d}, J$ $\left.=10.8 \mathrm{~Hz}, 1 \mathrm{H}, \mathrm{CH}_{2} \mathrm{O}\right), 2.25\left(\mathrm{~d}, J=13.5 \mathrm{~Hz}, 1 \mathrm{H}, \mathrm{CH}_{2}\right), 1.86\left(\mathrm{~d}, J=13.6 \mathrm{~Hz}, 1 \mathrm{H}, \mathrm{CH}_{2}\right), 1.83-$ $0.75\left(\mathrm{~m}, 13 \mathrm{H}, \mathrm{Cy}-\mathrm{H}+\mathrm{CH}_{2}\right), 1.71\left(\mathrm{~s}, 3 \mathrm{H}, \mathrm{CH}_{3}\right), 1.42\left(\mathrm{~s}, 3 \mathrm{H}, \mathrm{CH}_{3}\right) .{ }^{13} \mathrm{C} \mathrm{NMR}\left(100 \mathrm{MHz}, \mathrm{C}_{6} \mathrm{D}_{6}\right)$ : 
$\delta 143.0,138.5,115.4,107.7,69.8,40.6,39.2,37.3,34.1,27.1,26.9,26.5,25.5,18.5$. HRMS calcd for $\mathrm{C}_{16} \mathrm{H}_{26} \mathrm{O}: 234.1984$. Found: 234.1989 . The optical purity of $22(\sim 23 \%$ ee) was determined by chiral GC analysis (CDGTA column, $20 \mathrm{psi}, 80{ }^{\circ} \mathrm{C}$; $\mathrm{t}_{\mathrm{r}}$ (major enantiomer) $=$ $312.3 \mathrm{~min}, \mathrm{t}_{\mathrm{r}}($ minor enantiomer $\left.)=317.5 \mathrm{~min}\right)$. Chromatograms are illustrated below:
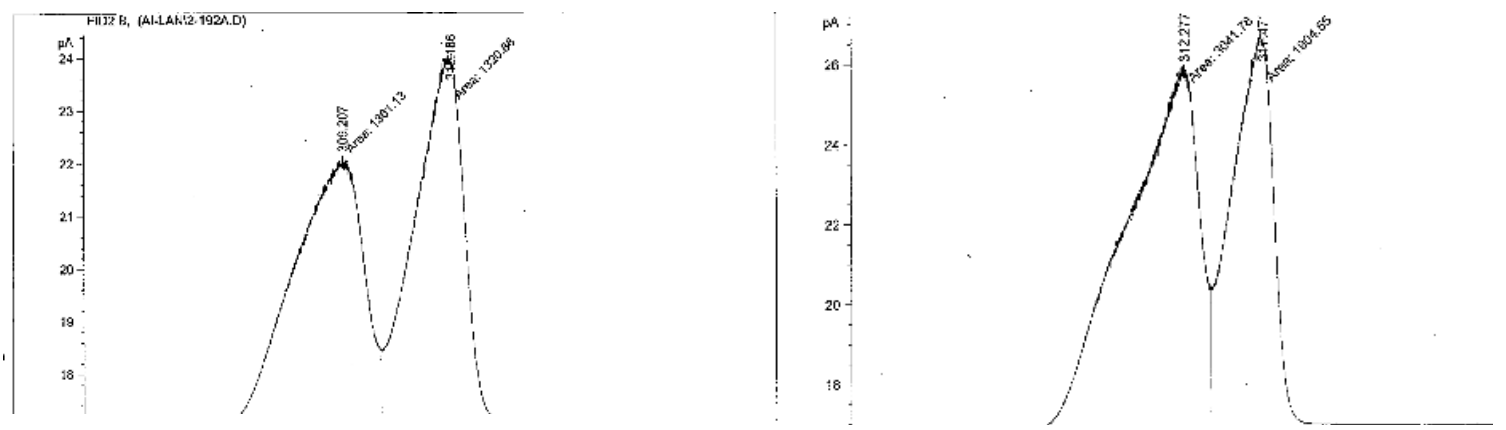

${ }^{1}$ H NMR spectrum of (R)-3-Cyclohexyl-5-methyl-3-(2-methylallyl)-3,4-dihydro-2H-pyran (22) illustrated below:

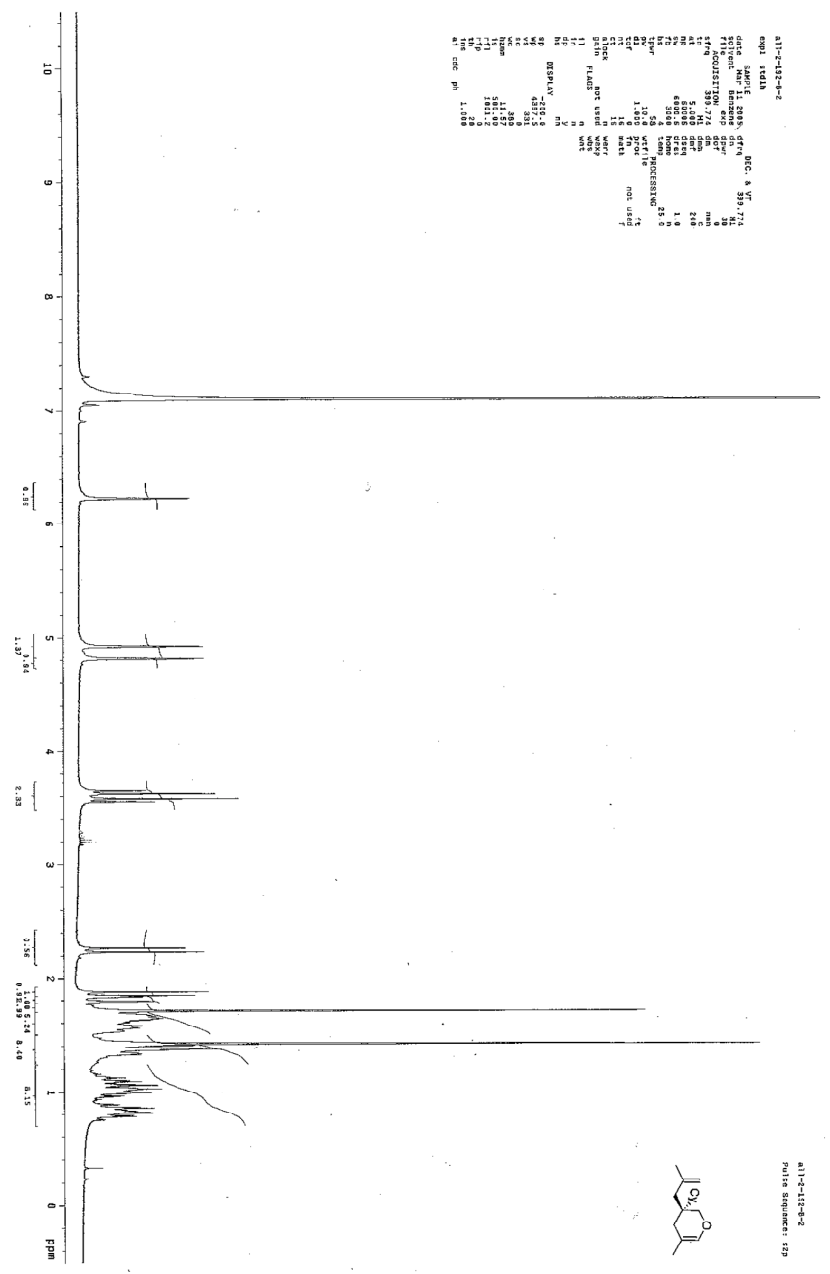


(S)-5-Methyl-3-(2-methylallyl)-3-phenyl-3,4-dihydro-2H-pyran (24a). $[\alpha]_{D}^{20}+0.014(c=$ 0.733, $\mathrm{CHCl}_{3}$ ). IR (neat): 2917 (s), 1680 (m), 1641 (w), 1446 (m), 1151 (s), 894 (w) cm ${ }^{-1} .{ }^{1} \mathrm{H}$ NMR (400 MHz, $\left.\mathrm{C}_{6} \mathrm{D}_{6}\right): \delta 7.10-6.95(\mathrm{~m}, 5 \mathrm{H}, \mathrm{Ar}-H), 6.16\left(\mathrm{q}, J=1.5 \mathrm{~Hz}, 1 \mathrm{H}, \mathrm{OCH}=\mathrm{CCH}_{3}\right)$, 4.75-4.73 (m, $\left.1 \mathrm{H}, \mathrm{CH}_{3} \mathrm{C}=\mathrm{CH} H^{\prime}\right), 4.60-4.59\left(\mathrm{~m}, 1 \mathrm{H}, \mathrm{CH}_{3} \mathrm{C}=\mathrm{CH} \mathrm{H}^{\prime}\right), 3.97(\mathrm{dd}, J=12.2,1.8 \mathrm{~Hz}$, 1H, CH$\left.H^{\prime} \mathrm{O}\right), 3.79\left(\mathrm{dd}, J=12.2,0.9 \mathrm{~Hz}, 1 \mathrm{H}, \mathrm{CH} \mathrm{H}^{\prime} \mathrm{O}\right), 2.37$ (d, $1 \mathrm{H}, J=13.3 \mathrm{~Hz}$, $\left.\mathrm{H}_{2} \mathrm{C}=\mathrm{CCH} H^{\prime}\right), 2.20\left(\mathrm{~d}, J=13.3 \mathrm{~Hz}, 1 \mathrm{H}, \mathrm{H}_{2} \mathrm{C}=\mathrm{CC} H \mathrm{H}^{\prime}\right), 2.11(\mathrm{~d}, J=16.7 \mathrm{~Hz}, 1 \mathrm{H}$, $\left.\mathrm{PhCCH} H^{\prime} \mathrm{C}=\mathrm{CHO}\right), 1.97\left(\mathrm{~d}, J=16.7 \mathrm{~Hz}, 1 \mathrm{H}, \mathrm{PhCC} H \mathrm{H}^{\prime} \mathrm{C}=\mathrm{CHO}\right), 1.42\left(\mathrm{~s}, 3 \mathrm{H}, \mathrm{OCH}=\mathrm{CCH}_{3}\right)$, $1.14\left(\mathrm{~s}, 3 \mathrm{H}, \mathrm{CH}_{3} \mathrm{C}=\mathrm{CH}_{2}\right) .{ }^{13} \mathrm{C} \mathrm{NMR}\left(100 \mathrm{MHz}, \mathrm{C}_{6} \mathrm{D}_{6}\right): \delta 144.9,142.2,138.3,128.2,126.4$, 126.3, 114.8, 106.7, 71.4, 45.7, 39.0, 37.0, 24.4, 18.2. HRMS calcd for $\mathrm{C}_{16} \mathrm{H}_{20} \mathrm{O}: 228.1514$. Found: 228.1514 . The optical purity of $\mathbf{2 4 a}(87 \%$ ee) was determined by chiral GC analysis $(\alpha-$ Dex column, $15 \mathrm{psi}, 100{ }^{\circ} \mathrm{C}$; $\mathrm{t}_{\mathrm{r}}($ minor enantiomer $)=359 \mathrm{~min}, \mathrm{t}_{\mathrm{r}}($ major enantiomer $)=365$ min). Chromatograms are illustrated below:
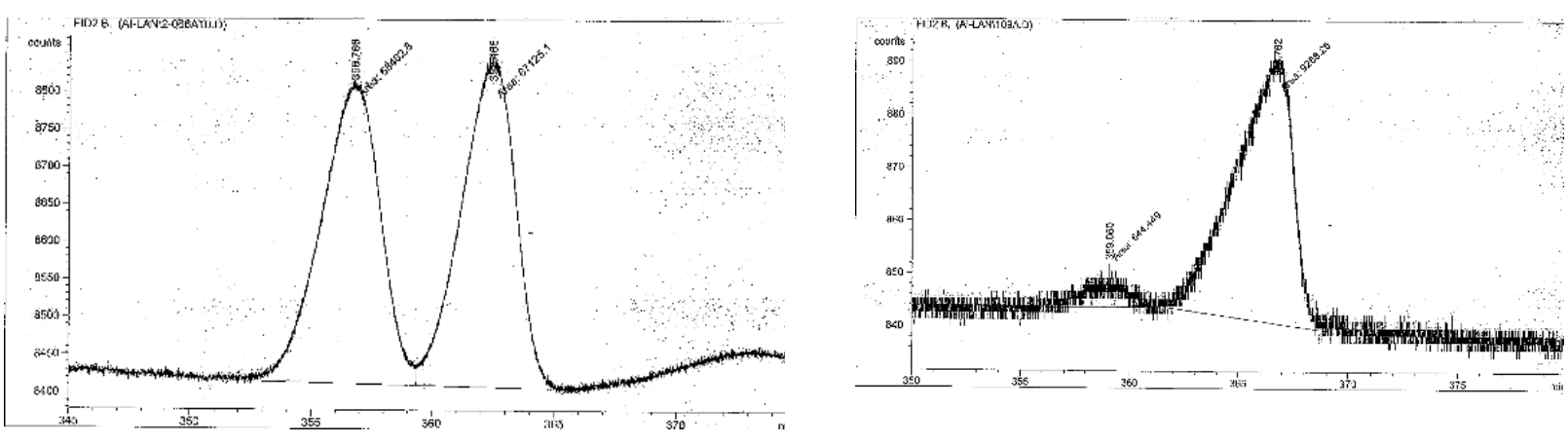

(S)-3-(4-Methoxyphenyl)-5-methyl-3-(2-methylallyl)-3,4-dihydro-2H-pyran (24b). $[\alpha]_{D}^{20}$

-27.5 (c = 0.98, $\left.\mathrm{CHCl}_{3}\right)$. IR (neat): $2961(\mathrm{w}), 2917(\mathrm{~m}), 2855(\mathrm{w}), 2836(\mathrm{w}), 1678(\mathrm{~m}), 1640$ (w), 1615 (m), 1583 (w), 1514 (s), 1464 (m), 1438 (m), 1294 (w), 1250 (s), 1187 (s), 1155 (s), 1036 (m), 1017 (m), 941 (w), 897 (w), $828(\mathrm{w}) \mathrm{cm}^{-1} .{ }^{1} \mathrm{H}$ NMR (400 MHz, CDCl $): \delta 7.18(\mathrm{~d}, J$ $=8.8 \mathrm{~Hz}, 2 \mathrm{H}, \operatorname{Ar}-H), 6.84(\mathrm{~d}, J=8.8 \mathrm{~Hz}, 2 \mathrm{H}, \operatorname{Ar}-H), 6.15(\mathrm{dd}, J=1.6,1.2 \mathrm{~Hz}, 1 \mathrm{H}$, $\left.\mathrm{OCH}=\mathrm{CCH}_{3}\right), 4.72\left(\mathrm{~s}, 1 \mathrm{H}, \mathrm{CH}_{3} \mathrm{C}=\mathrm{CH} H^{\prime}\right), 4.49\left(\mathrm{~s}, 1 \mathrm{H}, \mathrm{CH}_{3} \mathrm{C}=\mathrm{C} H \mathrm{H}^{\prime}\right), 3.98$ (dd, $J=10.4,1.6$ Hz, 1H, OCH H'CAr), 3.90 (dd, $\left.J=10.4,1.2 \mathrm{~Hz}, 1 \mathrm{H}, \mathrm{OCHH}{ }^{\prime} \mathrm{CAr}\right), 3.78$ (s, 3H, OCH $H_{3}, 2.41$ $\left(\mathrm{d}, J=13.2 \mathrm{~Hz}, 1 \mathrm{H}, \mathrm{CH} H^{\prime} \mathrm{C}=\mathrm{CH}_{2}\right), 2.30\left(\mathrm{~d}, J=16.8 \mathrm{~Hz}, 1 \mathrm{H}, \mathrm{C} H \mathrm{H}^{\prime} \mathrm{C}=\mathrm{CHO}\right), 2.29(\mathrm{~d}, J=13.2$ $\left.\mathrm{Hz}, 1 \mathrm{H}, \mathrm{CH} H^{\prime} \mathrm{C}=\mathrm{CH}_{2}\right), 2.17\left(\mathrm{~d}, J=16.8 \mathrm{~Hz}, 1 \mathrm{H}, \mathrm{CH} \mathrm{H}^{\prime} \mathrm{C}=\mathrm{CHO}\right), 1.60\left(\mathrm{~s}, 3 \mathrm{H}, \mathrm{OCH}=\mathrm{CCH}_{3}\right)$, $1.20\left(\mathrm{~s}, 3 \mathrm{H}, \mathrm{CH}_{3} \mathrm{C}=\mathrm{CH}_{2}\right) .{ }^{13} \mathrm{C} \mathrm{NMR}\left(100 \mathrm{MHz}, \mathrm{CDCl}_{3}\right): \delta 158.1,142.5,137.8,136.6,127.5$, 114.8, 113.7, 107.5, 72.1, 55.4, 46.1, 38.6, 36.8, 24.6, 18.5. HRMS calcd for $\mathrm{C}_{17} \mathrm{H}_{22} \mathrm{O}_{2}$ : 258.1620. Found: 258.1616. The optical purity of $\mathbf{2 4 b}$ ( $85 \%$ ee) was determined by chiral 
HPLC analysis (CHIRALPAK AS column, 100\% hexane, $1 \mathrm{~mL} / \mathrm{min}, 210 \mathrm{~nm}$; $\mathrm{t}_{\mathrm{r}}$ (major enantiomer $)=8.6 \mathrm{~min}, \mathrm{t}_{\mathrm{r}}($ minor enantiomer $\left.)=10.3 \mathrm{~min}\right)$. Chromatograms are illustrated below:
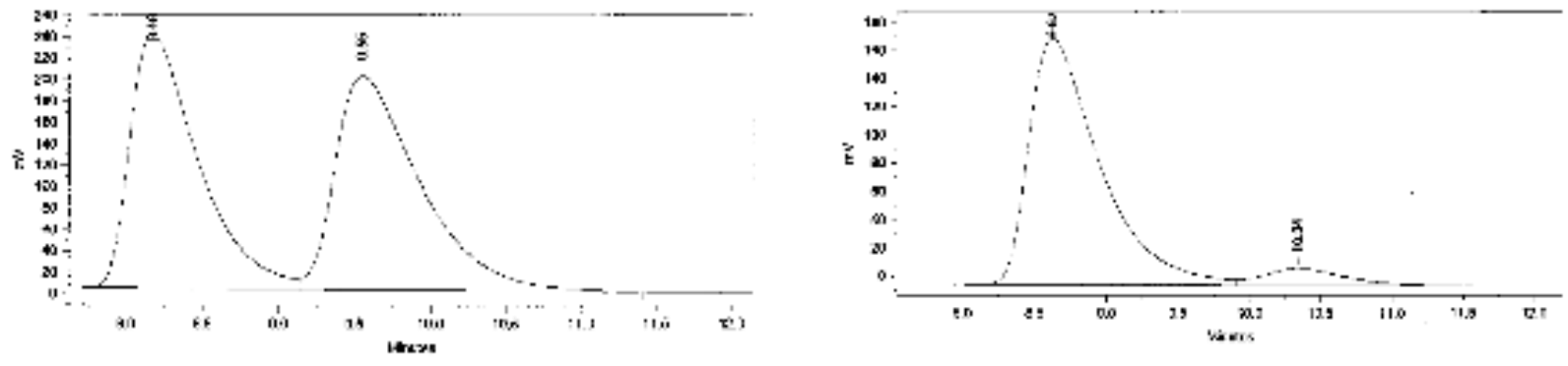

(S)-3-(4-Bromophenyl)-5-methyl-3-(2-methylallyl)-3,4-dihydro-2H-pyran (24c). $[\alpha]_{D}^{20}$

-30.4 (c = 1.0, $\left.\mathrm{CHCl}_{3}\right)$. IR (neat): $2962(\mathrm{~m}), 2911(\mathrm{~s}), 2880(\mathrm{~m}), 2855(\mathrm{~m}), 1684(\mathrm{~m}), 1640(\mathrm{w})$, 1495 (s), 1457 (m), 1438 (m), 1376 (w), 1155 (s), 1080 (m), 1011 (s), 941 (w), 891 (m), 822 (m) $\mathrm{cm}^{-1} .{ }^{1} \mathrm{H}$ NMR (400 MHz, $\left.\mathrm{CDCl}_{3}\right): \delta 7.42(\mathrm{~d}, J=6.4 \mathrm{~Hz}, 2 \mathrm{H}, \mathrm{Ar}-H), 7.15(\mathrm{~d}, J=6.4 \mathrm{~Hz}$, $2 \mathrm{H}, \mathrm{Ar}-H), 6.13\left(\mathrm{~s}, 1 \mathrm{H}, \mathrm{OCH}=\mathrm{CCH}_{3}\right), 4.74\left(\mathrm{~s}, 1 \mathrm{H}, \mathrm{CH}_{3} \mathrm{C}=\mathrm{CH} H^{\prime}\right), 4.50\left(\mathrm{~s}, 1 \mathrm{H}, \mathrm{CH}_{3} \mathrm{C}=\mathrm{CHH}^{\prime}\right)$, $3.94\left(\mathrm{~s}, 2 \mathrm{H}, \mathrm{CH}_{2} \mathrm{O}\right), 2.42\left(\mathrm{~d}, J=13.6 \mathrm{~Hz}, 1 \mathrm{H}, \mathrm{CH} H^{\prime} \mathrm{C}=\mathrm{CH}_{2}\right), 2.28(\mathrm{~d}, J=13.6 \mathrm{~Hz}, 1 \mathrm{H}$,

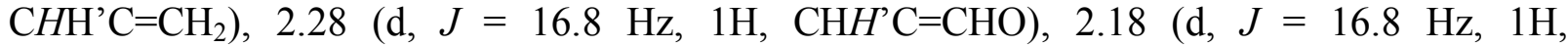
$\left.\mathrm{CH} \mathrm{H}^{\prime} \mathrm{C}=\mathrm{CHO}\right), 1.59\left(\mathrm{~s}, 3 \mathrm{H}, \mathrm{OCH}=\mathrm{CCH}_{3}\right), 1.23\left(\mathrm{~s}, 3 \mathrm{H}, \mathrm{CH}_{3} \mathrm{C}=\mathrm{CH}_{2}\right) .{ }^{13} \mathrm{C} \mathrm{NMR}(100 \mathrm{MHz}$, $\left.\mathrm{CDCl}_{3}\right): \delta 143.6,141.8,138.0,131.5,128.4,120.4,115.3,107.3,71.8,46.2,39.2,36.8,24.7$, 18.5. HRMS calcd for $\mathrm{C}_{16} \mathrm{H}_{19} \mathrm{OBr}$ : 306.0619. Found: 306.0605 . The optical purity of $24 \mathrm{c}(83 \%$ ee) was determined by chiral HPLC analysis (OD column, 100\% hexane, $1 \mathrm{~mL} / \mathrm{min}, 210 \mathrm{~nm}$; $\mathrm{t}_{\mathrm{r}}$ $($ major enantiomer $)=8.0 \mathrm{~min}, \mathrm{t}_{\mathrm{r}}($ minor enantiomer $\left.)=8.9 \mathrm{~min}\right)$. Chromatograms are illustrated below:
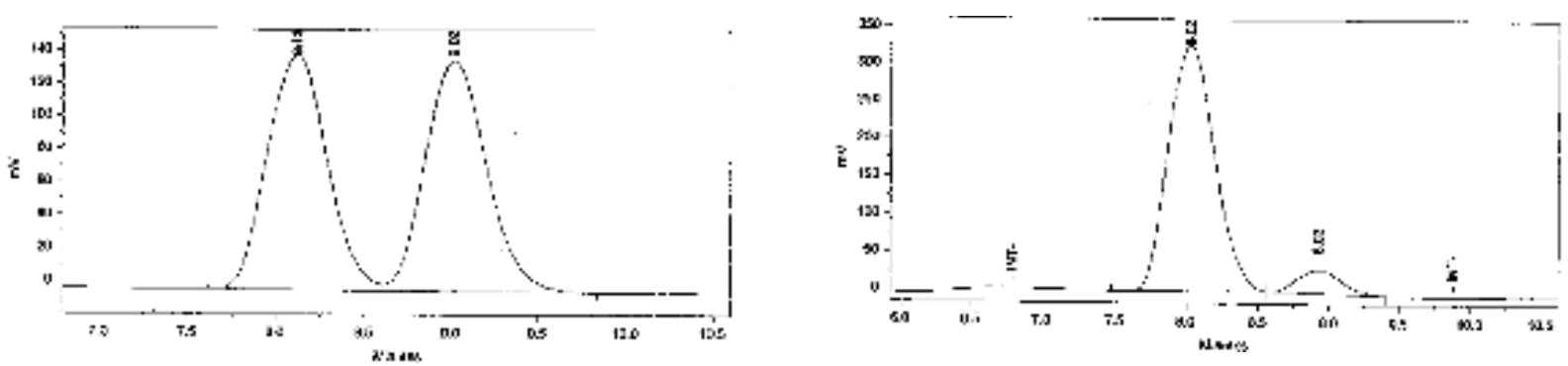
(R)-Methyl 5-methyl-3-(2-methylallyl)-3,4-dihydro-2H-pyran-3-carboxylate $(\mathbf{2 6}) . \quad[\alpha]_{D}^{20}$ -32.2 (c = 0.513, $\left.\mathrm{CHCl}_{3}\right)$. IR (neat): $2955(\mathrm{~m}), 2911(\mathrm{~m}), 1741(\mathrm{~s}), 1678(\mathrm{w}), 1445(\mathrm{~m}), 1199$ (s), 1149 (s), 1049 (w), 1023 (w) cm ${ }^{-1} .{ }^{1} \mathrm{H}$ NMR (400 MHz, $\left.\mathrm{C}_{6} \mathrm{D}_{6}\right)$ : $\delta 6.15$ (s, 1H, $\left.\mathrm{OCH}=\mathrm{CCH}_{3}\right), 4.78\left(\mathrm{~s}, 1 \mathrm{H}, \mathrm{CH}_{3} \mathrm{C}=\mathrm{CH} H^{\prime}\right), 4.70\left(\mathrm{~s}, 1 \mathrm{H}, \mathrm{CH}_{3} \mathrm{C}=C H \mathrm{H}^{\prime}\right), 4.00(\mathrm{~d}, J=10.4 \mathrm{~Hz}, 1 \mathrm{H}$, $\mathrm{CO} 2 \mathrm{MeCCH} H^{\prime} \mathrm{O}$ ), 3.67 (d, $\left.J=10.4 \mathrm{~Hz}, 1 \mathrm{H}, \mathrm{CO} 2 \mathrm{MeCC} H \mathrm{H}^{\prime} \mathrm{O}\right), 3.24$ (s, 3H, $\mathrm{CO}_{2} \mathrm{CH}_{3}$ ), 2.36 (d, $\left.J=13.5 \mathrm{~Hz}, 1 \mathrm{H}, \mathrm{CH} H^{\prime}\right), 2.36\left(\mathrm{~d}, J=16.9 \mathrm{~Hz}, 1 \mathrm{H}, \mathrm{CH} H^{\prime}\right), 2.04$ (d, $\left.J=13.5 \mathrm{~Hz}, 1 \mathrm{H}, \mathrm{CH} \mathrm{H}^{\prime}\right)$, $1.73\left(\mathrm{~d}, J=16.9 \mathrm{~Hz}, 1 \mathrm{H}, \mathrm{CH} \mathrm{H}^{\prime}\right), 1.55\left(\mathrm{~s}, 3 \mathrm{H}, \mathrm{OCH}=\mathrm{CCH}_{3}\right), 1.10\left(\mathrm{~s}, 3 \mathrm{H}, \mathrm{CH}_{3} \mathrm{C}=\mathrm{CH}_{2}\right) .{ }^{13} \mathrm{C}$ NMR (100 MHz, $\left.\mathrm{C}_{6} \mathrm{D}_{6}\right): \delta 174.4,141.1,138.3,115.0,107.2,69.4,51.2,44.6,42.7,33.9,23.3$, 18.0. HRMS calcd for $\mathrm{C}_{12} \mathrm{H}_{18} \mathrm{O}_{3}: 210.1256$. Found 210.1251 . Optical purity of $\mathbf{2 6}$ (94\% ee) was determined by chiral GC analysis (CDGTA column, $15 \mathrm{psi}, 70{ }^{\circ} \mathrm{C}$; $\mathrm{t}_{\mathrm{r}}$ (minor enantiomer) $=$ $189 \mathrm{~min}, \mathrm{t}_{\mathrm{r}}($ major enantiomer $\left.)=191 \mathrm{~min}\right)$. Chromatograms are illustrated below:
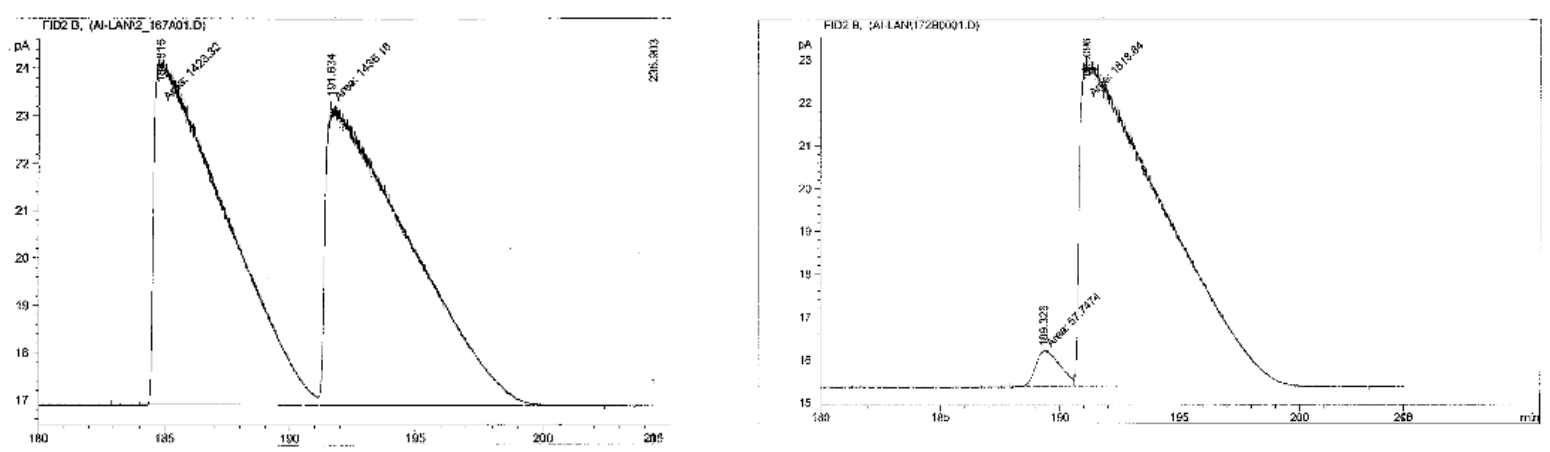

(R)-Benzyl-5-methyl-3-(2-methylallyl)-3,4-dihydro-2H-pyran-3-ylcarbamate $\quad(28) . \quad[\alpha]_{D}^{20}$ -26.1 (c = 0.367, $\mathrm{CHCl}_{3}$ ). IR (neat): 3345 (br), 2955 (m), 2924 (s), $2854(\mathrm{~m}), 1722$ (s), 1678 (m), 1646 (w), 1508 (s), 1451 (m), 1438 (w), 1376 (w), 1262 (s), 1231 (s), 1073 (s), 1029 (m) $\mathrm{cm}^{-1} .{ }^{1} \mathrm{H}$ NMR (400 MHz, $\left.\mathrm{CDCl}_{3}\right): \delta 7.35-7.27(\mathrm{~m}, 5 \mathrm{H}, \mathrm{Ar}-H), 6.17\left(\mathrm{~s}, 1 \mathrm{H}, \mathrm{OCH}=\mathrm{CCH}_{3}\right), 5.05$ (s, 2H, $\left.\mathrm{PhCH}_{2}\right), 4.87\left(\mathrm{~s}, 1 \mathrm{H}, \mathrm{CH}_{3} \mathrm{C}=\mathrm{CH} H^{\prime}\right), 4.76$ (br s, 1H, $\left.\mathrm{NH}\right), 4.67\left(\mathrm{~s}, 1 \mathrm{H}, \mathrm{CH}_{3} \mathrm{C}=\mathrm{CHH}^{\prime}\right)$, $4.11\left(\mathrm{~d}, J=10.4 \mathrm{~Hz}, 1 \mathrm{H}, \mathrm{OCH} H^{\prime}\right), 3.33$ (d, $\left.J=10.4 \mathrm{~Hz}, 1 \mathrm{H}, \mathrm{OCH} \mathrm{H}^{\prime}\right), 2.59$ (d, $J=13.2 \mathrm{~Hz}$, $\left.1 \mathrm{H}, \mathrm{CH} H^{\prime} \mathrm{C}=\mathrm{CH}_{2}\right), 2.45\left(\mathrm{~d}, J=13.2 \mathrm{~Hz}, 1 \mathrm{H}, \mathrm{CH} \mathrm{H}^{\prime} \mathrm{C}=\mathrm{CH}_{2}\right), 2.12(\mathrm{~d}, J=17.2 \mathrm{~Hz}, 1 \mathrm{H}$, $\left.\mathrm{CH} H^{\prime} \mathrm{C}=\mathrm{CHO}\right), 2.05\left(\mathrm{~d}, J=17.2 \mathrm{~Hz}, 1 \mathrm{H}, \mathrm{CH} \mathrm{H}^{\prime} \mathrm{C}=\mathrm{CHO}\right), 1.74\left(\mathrm{~s}, 3 \mathrm{H}, \mathrm{CH}_{3}\right), 1.53\left(\mathrm{~s}, 3 \mathrm{H}, \mathrm{CH}_{3}\right)$. ${ }^{13} \mathrm{C}$ NMR (100 MHz, $\left.\mathrm{CDCl}_{3}\right): \delta 141.1,138.0,136.8,128.7,128.3,128.2,115.8,106.7,69.2$, 66.5, 52.0, 41.5, 36.7, 24.6, 18.2. HRMS calcd for $\mathrm{C}_{18} \mathrm{H}_{23} \mathrm{NO}_{3}$ : 301.1678. Found: 301.1681. The optical purity of $\mathbf{2 8}$ (54\% ee) was determined by chiral HPLC analysis (CHIRALCEL OD column, 95:5 hexanes: $i-\mathrm{PrOH}, 1 \mathrm{~mL} / \mathrm{min}, 210 \mathrm{~nm}$; $\mathrm{t}_{\mathrm{r}}$ (minor enantiomer) $=7.6 \mathrm{~min}, \mathrm{t}_{\mathrm{r}}$ (major enantiomer $)=10.4 \mathrm{~min})$. Chromatograms are illustrated below: 
Lee, et al., Page S13
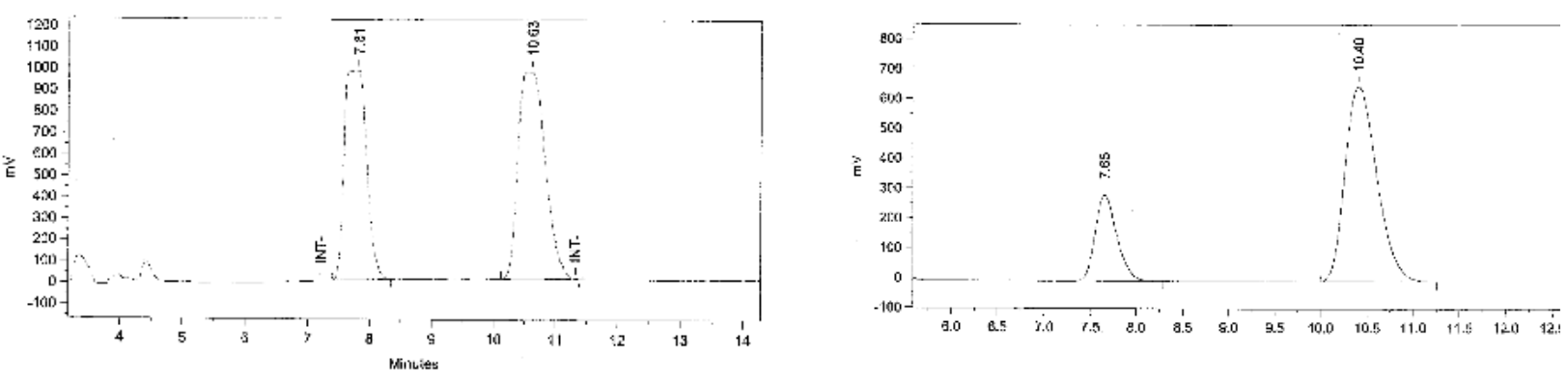


\section{Representative data from catalyst screening:}

\begin{tabular}{|c|c|c|c|c|c|}
\hline catalyst & mol \% & temp. $\left({ }^{\circ} \mathrm{C}\right)$ & time $(\mathrm{h})$ & conv. $(\%)^{\mathrm{a}}$ & ee $(\%)^{b}$ \\
\hline 1 & 5 & 22 & 1 & $>98$ & - \\
\hline $\mathbf{3 a}$ & 10 & 22 & 21 & $<5$ & nd \\
\hline $3 \mathbf{b}$ & 10 & 22 & 21 & $<10$ & nd \\
\hline $3 c$ & 10 & 22 & 21 & $<5$ & nd \\
\hline $4 a$ & 10 & 22 & 21 & 56 & 86 \\
\hline $4 a$ & 10 & 60 & 21 & 64 & 80 \\
\hline $4 a$ & 15 & 22 & 21 & $>98$ & 87 \\
\hline $4 a$ & $15\left(\mathrm{Et}_{2} \mathrm{O}\right)$ & 22 & 21 & $>98$ & 87 \\
\hline $4 a$ & 15 (n-pentane) & 22 & 21 & $>98$ & 82 \\
\hline $4 c$ & 10 & 22 & 21 & $<5$ & nd \\
\hline 5 & 10 & 22 & 21 & $<5$ & nd \\
\hline 6 & 10 & 22 & 21 & $<5$ & nd \\
\hline $7 a$ & 5 & 65 & 4 & 85 & 9 \\
\hline $7 b$ & 5 & 65 & 4 & $>98$ & $<5$ \\
\hline $8 b$ & 5 & 65 & 12 & 94 & $<5$ \\
\hline $8 b$ & 5 & 22 & 12 & 13 & $<5$ \\
\hline
\end{tabular}

${ }^{\mathrm{a}}$ Determined by $400 \mathrm{MHz}{ }^{1} \mathrm{H}-\mathrm{NMR}$ analysis.

${ }^{\mathrm{b}}$ Determined by chiral GLC analysis $(\alpha$-dex $)$; nd $=$ not determined. 
(S)-3-Methyl-6-(3-methylbut-3-enyl)tetrahydro-2H-pyran-2-one (30). A solution of enol ether $14(25 \mathrm{mg}, 0.15 \mathrm{mmol})$ was dissolved in THF $(2.0 \mathrm{~mL})$ and $1 \mathrm{M} \mathrm{HCl}(0.50 \mathrm{~mL})$. The mixture was allowed to stir at $22{ }^{\circ} \mathrm{C}$ for $24 \mathrm{~h}$ before $\mathrm{Et}_{2} \mathrm{O}$ and water were added. The aqueous layer was washed 3 times with $\mathrm{Et}_{2} \mathrm{O}$, the combined organic layers were washed with brine and dried $\left(\mathrm{MgSO}_{4}\right)$. Silica gel chromatography (5:1 pentane:Et $\left.{ }_{2} \mathrm{O}\right)$ afforded a mixture of lactol diastereomers as a colorless oil $(21.5 \mathrm{mg}, 0.117 \mathrm{mmol}, 76 \%)$. A portion of the lactol mixture (14 mg, $0.066 \mathrm{mmol}, 0.076 \mathrm{mmol})$ was dissolved in dichloromethane $(0.4 \mathrm{~mL})$ and $4 \AA$ molecular sieves, NMO (13 mg, $0.11 \mathrm{mmol})$ and TPAP $(1.3 \mathrm{mg}, 3.8 \mu \mathrm{mol})$ were added successively. The mixture was allowed to stir at $22{ }^{\circ} \mathrm{C}$ for $2 \mathrm{~h}$ before it was concentrated by purging with a stream of nitrogen. Silica gel chromatography $\left(\mathrm{CH}_{2} \mathrm{Cl}_{2}\right)$ afforded the product 30 as a 1:1 mixture of diastereomers (12 mg, $0.067 \mathrm{mmol}, 88 \%$ ). IR (neat): $2936(\mathrm{~m}), 1741$ (s), 1646 (w), 1451 (w), 1376 (w), 1237 (w), 1168 (m), 1092 (m), $1017(\mathrm{w}), 891(\mathrm{w}) \mathrm{cm}^{-1} .{ }^{1} \mathrm{H}$ NMR (400 MHz, $\left.\mathrm{CDCl}_{3}\right): \delta 4.72(\mathrm{~s}, 1 \mathrm{H}$ x 2, C=CHH), $4.68(\mathrm{~s}, 1 \mathrm{H}$ x 2, C=CHH), 4.32-4.22 (m, $1 \mathrm{H} \times 2, \mathrm{CHOC}=\mathrm{O}), 2.64-2.54\left(\mathrm{~m}, 1 \mathrm{H}, \mathrm{CH}_{3} \mathrm{CHC}=\mathrm{O}\right.$-diastereomer 1$), 2.48-2.38(\mathrm{~m}, 1 \mathrm{H}$, $\mathrm{CH}_{3} \mathrm{CHC}=\mathrm{O}$-diastereomer 2), 2.26-1.46 (m, $\left.8 \mathrm{H} \mathrm{x} \mathrm{2,} \mathrm{CH}_{2}\right), 1.71\left(\mathrm{~s}, 3 \mathrm{H} \times 2, \mathrm{H}_{2} \mathrm{C}=\mathrm{CCH}_{3}\right), 1.29$ (d, 3H, J=6.8 Hz, CHC=O-diastereomer 1), 1.21 (d, 3H, J=6.8 Hz, CHC=O-diastereomer 2). ${ }^{13} \mathrm{C}$ NMR (100 MHz, $\left.\mathrm{CDCl}_{3}\right): \delta 176.6,174.5,144.9,144.8,110.7,110.6,81.5,77.7,36.3,34.4$, 33.9, 33.4, 33.3, 33.0, 29.3, 28.7, 26.9, 25.8, 22.7, 22.6, 17.6, 16.3. HRMS calcd for $\mathrm{C}_{11} \mathrm{H}_{18} \mathrm{O}_{2}$ : 182.1307. Found: 182.1303.

3-Hydroxy-3-methyl-6-(3-methylbut-3-enyl)tetrahydro-2H-pyran-2-one (31). A flask was charged with $t$ - $\mathrm{BuOH}(0.15 \mathrm{~mL})$ and water $(0.15 \mathrm{~mL}) . \quad \mathrm{AD}-\mathrm{mix}-\beta(42 \mathrm{mg})$ and methanesulfonamide $(3.0 \mathrm{mg}, 0.030 \mathrm{mmol})$ were added. The suspension was stirred at $22{ }^{\circ} \mathrm{C}$, then cooled to $0{ }^{\circ} \mathrm{C}$. Enol ether $14(5.0 \mathrm{mg}, 0.030 \mathrm{mmol})$ was added in $t$-BuOH $(0.20 \mathrm{~mL})$ and water $(0.20 \mathrm{~mL})$. The resulting suspension was allowed to stir at $0{ }^{\circ} \mathrm{C}$ for $8 \mathrm{~h}$ followed by the addition of sodium sulfite $(50 \mathrm{mg})$. The aqueous layer was washed 3 times with ethyl acetate, the combined organic layers were washed with a $2 \mathrm{~N}$ solution of $\mathrm{KOH}$ and dried $\left(\mathrm{MgSO}_{4}\right)$. Silica gel chromatography $\left(2: 1 \mathrm{Et}_{2} \mathrm{O}\right.$ :hexanes) afforded the hemiacetal diastereomers as a colorless oil (4.5 mg, $0.022 \mathrm{mmol}, 75 \%)$. A portion of this mixture (2.2 $\mathrm{mg}, 0.011 \mathrm{mmol})$ was dissolved in dichloromethane $(0.50 \mathrm{~mL})$ and treated with Dess-Martin periodinane $(6.4 \mathrm{mg}$, $0.015 \mathrm{mmol}$ ) at $22^{\circ} \mathrm{C}$ for $2 \mathrm{~h}$. The reaction was quenched by addition of a solution of sodium thiosulfate and diluted with water. The aqueous layer was washed 3 times with dichloromethane, the combined organic layers were washed with brine and dried $\left(\mathrm{MgSO}_{4}\right)$. 
Silica gel chromatography $\left(2: 1\right.$ pentane: $\left.\mathrm{Et}_{2} \mathrm{O}\right)$ afforded the product as a colorless oil $(2.0 \mathrm{mg}$, 0.010 mmol, 92\%). $[\alpha]_{D}^{20}-5.5\left(c=0.167, \mathrm{CHCl}_{3}\right)$. IR (neat): $3396(\mathrm{br}), 2917(\mathrm{~m}), 1722(\mathrm{~s}) \mathrm{cm}^{-}$ 1. ${ }^{1} \mathrm{H}$ NMR (400 MHz, $\left.\mathrm{CDCl}_{3}\right): \delta 8.08(\mathrm{~s}, 1 \mathrm{H}, O H), 5.03-4.95(\mathrm{~m}, 1 \mathrm{H}, \mathrm{CHOC}=\mathrm{O}), 4.71(\mathrm{~s}, 1 \mathrm{H}$, $\left.\mathrm{C}=\mathrm{CH} H^{\prime}\right), 4.66\left(\mathrm{~s}, 1 \mathrm{H}, \mathrm{C}=\mathrm{C} H \mathrm{H}^{\prime}\right), 2.48\left(\mathrm{~d}, J=7.6 \mathrm{~Hz}, 1 \mathrm{H}, \mathrm{CH}_{2}=\mathrm{CCH} H^{\prime}\right), 2.46(\mathrm{~d}, J=7.6 \mathrm{~Hz}$, $\left.1 \mathrm{H}, \mathrm{CH}_{2}=\mathrm{CCHH}^{\prime}\right), 2.13\left(\mathrm{~s}, 3 \mathrm{H}, \mathrm{OHCCH}_{3}\right), 2.05-1.90\left(\mathrm{~m}, 3 \mathrm{H}, \mathrm{CH}_{2}\right), 1.84-1.64\left(\mathrm{~m}, 3 \mathrm{H}, \mathrm{CH}_{2}\right)$, $1.70\left(\mathrm{~s}, 3 \mathrm{H}, \mathrm{CH}_{3} \mathrm{C}=\mathrm{C}\right) .{ }^{13} \mathrm{C} \mathrm{NMR}\left(100 \mathrm{MHz}, \mathrm{CDCl}_{3}\right): \delta 161.1,144.7,110.7,76.9,73.4,39.4$, 33.5, 32.4, 30.2, 28.0, 22.6. HRMS calcd for $\left[\mathrm{C}_{11} \mathrm{H}_{18} \mathrm{O}_{3}\right]+\mathrm{H}: 199.1334$. Found: 199.1340.

Proof of stereochemical identity of $13{ }^{8}$ As illustrated below, optically enriched 13, obtained from ARCM in the presence of (R)-4a, was selectively dihydroxylated at the enol ether using AD-mix- $\beta$. The diol was oxidatively cleaved with sodium periodate and the resulting formate was treated with a half-saturated solution of ammonia in methanol to yield the desired alcohol. Catalytic hydrogenation in the presence of $\mathrm{Pd} / \mathrm{C}$ provided the $\beta$-hydroxy ketone 32. The chiral GLC trace of 32, prepared through the method described above, was compared to the chiral GLC traces of $(S)$-32 obtained from (L)-proline-catalyzed enantioslective aldol reaction of acetone and isovaleraldehyde ${ }^{9}$ and $(S)$-32 made previously in these laboratories, ${ }^{10}$ and was found to be of the opposite configuration. The stereochemical identity of $\mathbf{1 3}$ formed under the conditions described through the use of $(R)-\mathbf{4 a}$ is $(R)-\mathbf{1 3}$.
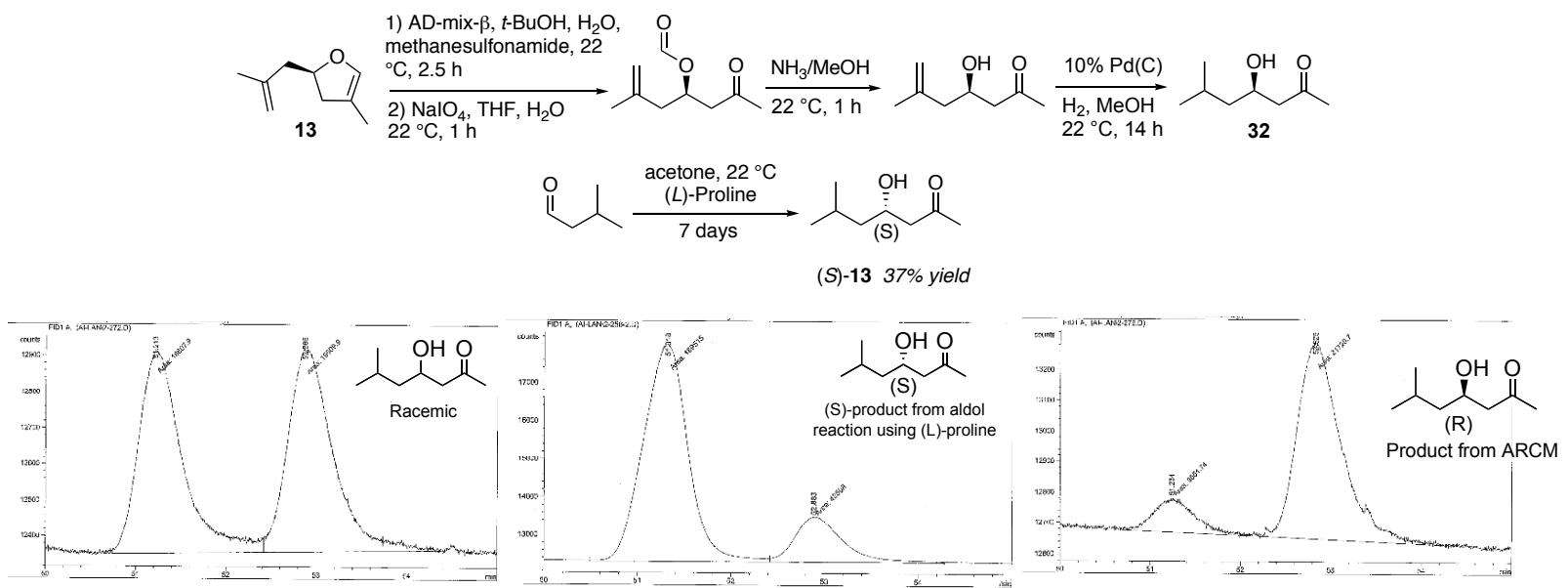

Proof of stereochemical identity of 26. ${ }^{11}$ DIBAL-H $(1.4 \mathrm{M}$ in hexanes, $0.11 \mathrm{ml}, 0.16 \mathrm{mmol})$ was added to a solution of the ester $26(11 \mathrm{mg}, 0.052 \mathrm{mmol})$ in $\mathrm{THF}(0.5 \mathrm{ml})$ at $-78{ }^{\circ} \mathrm{C}$. The

8) Stereochemical assignments for 14, 16, 18 and $\mathbf{2 0}$ are inferred from this correlation. It is important to note, however, that subtle variations can lead to reversal of the identity of the major product enantiomer. In case of application to total synthesis, rigorous determination of the identity of the major enantiomer is thus warranted.

9) B. List, P. Pojarliev, C. Castello, Org. Lett. 2001, 3, 573-575.

10) See the Supporting Information in A. F. Kiely, J. A. Jernelius, R. R. Schrock, A. H. Hoveyda, J. Am. Chem. Soc. 2002, 124, 2868-2869. 
mixture was allowed to stir at $-78{ }^{\circ} \mathrm{C}$ for $1 \mathrm{~h}$ before it was slowly warmed to $22{ }^{\circ} \mathrm{C}$. After an hour, the reaction was quenched by addition of an aqueous solution of Rochelle's salt. The aqueous layer was washed three times with $\mathrm{Et}_{2} \mathrm{O}$; the combined organic extracts were washed with brine and dried $\left(\mathrm{MgSO}_{4}\right)$. Silica gel chromatography $\left(2: 1\right.$ pentane: $\left.\mathrm{Et}_{2} \mathrm{O}\right)$ afforded the alcohol as a colorless oil $(8.5 \mathrm{mg}, 0.047 \mathrm{mmol}, 89 \%$ yield). A portion of this alcohol (4.5 $\mathrm{mg}$, $0.025 \mathrm{mmol})$ was dissolved in dichloromethane $(0.3 \mathrm{ml})$. Triethylamine $(10 \mu \mathrm{L}, 0.07 \mathrm{mmol})$, DMAP (1.2 mg, $0.001 \mathrm{mmol})$ and $p$-bromo benzoyl chloride $(6.5 \mathrm{mg}, 0.03 \mathrm{mmol})$ were added successively at $22{ }^{\circ} \mathrm{C}$. The mixture was allowed to stir at $22{ }^{\circ} \mathrm{C}$ for $3 \mathrm{~h}$ before it was diluted with dichloromethane and a saturated aqueous solution of sodium bicarbonate. The aqueous layer was washed with dichloromethane and dried $\left(\mathrm{MgSO}_{4}\right)$. Silica gel chromatography (13:1 hexanes:EtOAc) afforded the ester $\mathbf{2 9}$ as a colorless oil that solidified to a white solid upon standing (8.2 mg, $0.022 \mathrm{mmol}, 90 \%$ yield).

X-ray quality crystals were grown by slow evaporation of $\mathrm{Et}_{2} \mathrm{O}$ at $4{ }^{\circ} \mathrm{C}$. $\mathrm{mp}=49-51{ }^{\circ} \mathrm{C}$ $\left(\mathrm{Et}_{2} \mathrm{O}\right) .[\alpha]_{D}^{20}-10.0\left(c=0.32, \mathrm{CHCl}_{3}\right)$. IR (neat): $2917(\mathrm{~m}), 1718(\mathrm{~s}), 1675(\mathrm{w}), 1595(\mathrm{~m}), 1675$ (w), 1271 (s), 1161 (m), 1124 (s), 1106 (s), 1014 (s), 892 (w), 849 (w), 763 (s) cm ${ }^{-1}$. ${ }^{1}$ H NMR
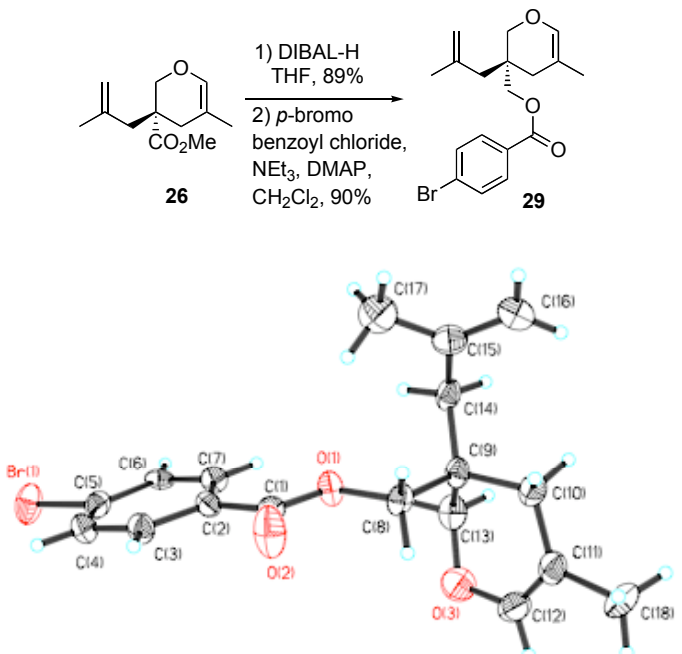

$\left(400 \mathrm{MHz}, \mathrm{CDCl}_{3}\right): \delta 7.88(\mathrm{~d}, J=8.0 \mathrm{~Hz}, 2 \mathrm{H}$, Ar-H), 7.58 (d, $J=8.0 \mathrm{~Hz}, 2 \mathrm{H}, \mathrm{Ar}-\mathrm{H}), 6.19$ (s, $\left.1 \mathrm{H}, \mathrm{OCH}=\mathrm{CCH}_{3}\right), 4.91\left(\mathrm{~s}, 1 \mathrm{H}, \mathrm{CH}_{3} \mathrm{C}=\mathrm{CH} H\right)$, $4.69\left(\mathrm{~s}, 1 \mathrm{H}, \mathrm{CH}_{3} \mathrm{C}=\mathrm{CHH}\right), 4.21(\mathrm{~d}, J=11.2 \mathrm{~Hz}$, $1 \mathrm{H}, \mathrm{CH} H \mathrm{OC}=\mathrm{O}), 4.16(\mathrm{~d}, J=11.2 \mathrm{~Hz}, 1 \mathrm{H}$, $\mathrm{CHHOC}=\mathrm{O}), 3.84(\mathrm{~d}, J=10.8 \mathrm{~Hz}, 1 \mathrm{H}$, $\mathrm{CH} H \mathrm{OCH}=\mathrm{C}), 3.61(\mathrm{~d}, J=10.8 \mathrm{~Hz}, 1 \mathrm{H}$, $\mathrm{CH} \mathrm{HOCH}=\mathrm{C}), 2.25\left(\mathrm{~d}, J=13.6 \mathrm{~Hz}, 1 \mathrm{H}, \mathrm{CH}_{2}\right)$, $2.11\left(\mathrm{~d}, J=13.6 \mathrm{~Hz}, 1 \mathrm{H}, \mathrm{CH}_{2}\right), 1.96(\mathrm{~d}, J=17.2$ $\left.\mathrm{Hz}, 1 \mathrm{H}, \mathrm{CH}_{2}\right), 1.80\left(\mathrm{~d}, J=17.2 \mathrm{~Hz}, 1 \mathrm{H}, \mathrm{CH}_{2}\right)$, $1.77\left(\mathrm{~s}, 3 \mathrm{H}, \mathrm{CH}_{3}\right), 1.55\left(\mathrm{~s}, 3 \mathrm{H}, \mathrm{CH}_{3}\right) .{ }^{13} \mathrm{C} \mathrm{NMR}\left(100 \mathrm{MHz}, \mathrm{CDCl}_{3}\right): \delta 165.9,141.1,137.9$, 132.0, 131.2, 129.3, 116.1, 107.1, 94.6, 69.5, 66.8, 41.3, 36.2, 33.9, 25.4, 18.4. HRMS calcd for $\mathrm{C}_{18} \mathrm{H}_{21} \mathrm{BrO}_{3}$ : 364.0674. Found: 364.0682 .

Crystal data: $\mathrm{C}_{18} \mathrm{H}_{21} \mathrm{BrO}_{3}, \mathrm{M}=365.26$, monoclinic, $a=18.560(4), b=5.8676(11), c=$ $15.887(3), b=98.155(4)^{\circ}, U=1712.6(6) \AA^{3}, T=192(3) \mathrm{K}$. space group $\mathrm{C} 2, Z=4, m=2.410$ mm-1, 6041 reflections collected, 3703 independent reflections $\left(\mathrm{R}_{\mathrm{int}}=0.0427\right)$. Flack parameter $=0.052(15)$. The final $w \mathrm{R}\left(\mathrm{F}^{2}\right)$ was 0.0856 . 